Pacific

Journal of

Mathematics

THE SCALAR CURVATURE DEFORMATION EQUATION ON LOCALLY CONFORMALLY FLAT MANIFOLDS OF HIGHER DIMENSIONS

YU YAN 


\title{
THE SCALAR CURVATURE DEFORMATION EQUATION ON LOCALLY CONFORMALLY FLAT MANIFOLDS OF HIGHER DIMENSIONS
}

\author{
YU YAN
}

\begin{abstract}
We study the equation $\Delta_{g} u-(n-2) /(4(n-1)) R(g) u+K u^{p}=0$ for $p$ in $1+\zeta \leq p \leq(n+2) /(n-2)$ on locally conformally flat compact manifolds $\left(M^{n}, g\right)$. We prove that when the scalar curvature $R(g) \equiv 0$ and $n \geq 5$, under suitable conditions on $K$, all positive solutions $u$ with bounded energy have uniform upper and lower bounds. In our previous 2007 paper, we also assumed this energy bound condition for the uniform estimates in the lowerdimensional case. We now give an example showing that this condition is necessary.
\end{abstract}

\section{Introduction}

Let $\left(M^{n}, g\right)$ be an $n$-dimensional compact manifold with metric $g$, and denote by $R(g)$ the scalar curvature of $g$. Let $u$ be a positive function defined on $M$. The scalar curvature of the conformally deformed metric $u^{4 /(n-2)} g$ is given by

$$
R\left(u^{4 /(n-2)} g\right)=-c(n)^{-1} u^{-(n+2) /(n-2)}\left(\Delta_{g} u-c(n) R(g) u\right),
$$

where $c(n)=(n-2) /(4(n-1))$.

The Yamabe theorem, which was proved by Trudinger [1968], Aubin [1976] and Schoen [1984], says that there exists a $u>0$ such that $R\left(u^{4 /(n-2)} g\right)$ is equal to some constant $K$. The PDE formulation of this theorem is that the equation

$$
\Delta_{g} u-c(n) R(g) u+c(n) K u^{(n+2) /(n-2)}=0
$$

has a positive solution for some constant $K$.

J. Escobar and R. Schoen [1986] extended this result to the case when $K$ is a function on $M$. They proved that, under certain conditions on $K$, the above equation has a positive solution $u$ when $R(g)>0$ or $R(g) \equiv 0$.

In fact, the solution in those existence results minimizes the associated constraint variational problem and can be obtained as a limit of a sequence of solutions of the corresponding subcritical equations. Therefore, a natural question is whether

MSC2000: 53C21.

Keywords: scalar curvature, conformal deformation, uniform estimates. 
nonminimal solutions can also be produced from solutions of the subcritical equations. We would like to know if there are uniform estimates for solutions of the equation

$$
\Delta_{g} u-c(n) R(g) u+K u^{p}=0, \quad \text { where } 1+\zeta \leq p \leq(n+2) /(n-2) .
$$

This was proved to be true by Schoen [1988a; 1991] when $K$ is a positive constant, $R(g)>0$, and $\left(M^{n}, g\right)$ is locally conformally flat and not conformally diffeomorphic to $S^{n}$. When the manifold has dimension $n=3$, the work of Y. Li and M. Zhu [1999] shows that the same estimates hold when the locally conformally flat condition on $M$ is dropped. This result was extended to dimensions $n=4,5$ by O. Druet [2003; 2004], and then to dimensions $n \leq 7$ independently by Y. Li and L. Zhang [Li and Zhang 2005] and F. C. Marques [2005]; when the dimension $n \geq 8$, it was proved by Li and Zhang [2005] under an additional assumption on the Weyl tensor of the backgroud metric $g$.

When $K$ is a positive function, Y. Li and M. Zhu [1999] obtained uniform estimates for the solutions when $(M, g)$ is a 3-dimensional compact manifold with $R(g)>0$ and is not conformally diffeomorphic to $S^{3}$. When the dimension $n \geq 4$, the same estimates hold on a locally conformally flat, scalar positive, compact manifold that is not conformally diffeomorphic to $S^{n}$, under the following additional flatness condition on $K$ : near each critical point of $K$, there exists a neighborhood and a constant $C_{0}$ such that in that neighborhood

$$
\left|\nabla^{p} K\right| \leq C_{0}|\nabla K|^{(n-2+\epsilon-p) /(n-3+\epsilon)} \quad \text { for } 2 \leq p \leq n-2,
$$

where $\epsilon>0$ and $\nabla^{p} K$ is the $p$-th covariant derivative of $K$. This result is proved by fine blow-up analysis similar to the analyses in [Li 1995] and [Schoen 1988a].

In [Yan 2007], we studied this problem on 3 and 4 dimensional locally conformally flat compact manifolds with zero scalar curvature. In this paper, we consider the higher-dimensional case $n \geq 5$.

When the scalar curvature $R(g) \equiv 0$ on the manifold $M$, Equation (1) becomes

$$
\Delta_{g} u+K u^{p}=0, \quad \text { where } 1+\zeta \leq p \leq(n+2) /(n-2) .
$$

The necessary conditions for the existence of a solution $u>0$ are that $K$ changes sign on $M$ and $\int_{M} K d v_{g}<0$.

The corresponding existence result is as follows.

Theorem 1.1 [Escobar and Schoen 1986]. Suppose $M$ is locally conformally flat with zero scalar curvature. Suppose $K$ is a nonzero smooth function on $M$ satisfying the condition that there is a maximum point $P_{0} \in M$ of $K$ at which all derivatives of $K$ of order less than or equal to $(n-3)$ vanish. Then $K$ is the scalar curvature of a metric $\bar{g}=u^{4 /(n-2)} g$ for some $u>0$ on $M$ if and only if $K$ is 
such that $K$ changes sign and $\int_{M} K d v_{g}<0$. When the dimension $n=3$ or 4 , the flatness condition on $K$ is automatically satisfied, and the locally conformally flat assumption on $M$ can be removed.

There is a compactness theorem when the dimension of $M$ is equal to 3 or 4 :

Theorem 1.2 [Yan 2007]. Let $(M, g)$ be a 3- or 4-dimensional locally conformally flat compact manifold with $R(g) \equiv 0$. Let $\mathscr{K}:=\left\{K \in C^{3}(M): K>0\right.$ somewhere on $M, \int_{M} K d v_{g} \leq-C_{K}{ }^{-1}<0$, and $\left.\|K\|_{C^{3}(M)} \leq C_{K}\right\}$ for some constant $C_{K}$, and let $S_{\Lambda}:=\left\{u: u>0\right.$ solves (2) with $K \in \mathscr{K}$, and $\left.E(u):=\int_{M}|\nabla u|^{2} d v_{g} \leq \Lambda\right\}$. Then there exists $C=C\left(M, g, C_{K}, \Lambda, \zeta\right)>0$ such that $u \in S_{\Lambda}$ satisfies $\|u\|_{C^{3}(M)} \leq C$ and $\min _{M} u \geq C^{-1}$.

In Section 2, we will give an example that shows that these estimates cannot be improved to be independent of the energy $E(u)$.

Next we give a similar theorem on manifolds of dimension $n \geq 5$. We first need to define a flatness condition on $K$ as follows.

Definition 1.3. A function $K \in C^{n-2}(M)$ is said to satisfy the flatness condition (*) if near each critical point $P$ of $K$ where $K(P)>0$, there exist a neighborhood and a constant $C_{0}$ such that, in that neighborhood,

$$
\left|\nabla^{p} K\right| \leq C_{0}|\nabla K|^{(n-2-p) /(n-3)} \text { for } 2 \leq p \leq n-3,
$$

where $\nabla^{p} K$ is the $p$-th covariant derivative of $K$.

In particular, this implies that all partial derivatives of $K$ up to order $n-3$ vanish at those critical points, and the order of flatness is the same as that in Theorem 1.1. A simple example of a function satisfying this condition is a function that can be expressed near the critical points as $K(z)=a+b|z|^{n-2}$, where $a$ and $b$ are constants and $z$ is a local coordinate system centered at the critical point. This type of flatness condition also appeared when Y. Li [1995; 1996] studied the problem of prescribing scalar curvature functions on $S^{n}$.

We are ready to state the theorem:

Theorem 1.4. Let $\left(M^{n}, g\right)$ be a locally conformally flat compact manifold with $R(g) \equiv 0$ and dimension $n \geq 5$. Let $K \in C^{n-2}(M)$ be a function satisfying the flatness condition (*); assume $K$ is positive somewhere on $M$ and $\int_{M} K d v_{g}<0$. If $u$ is a positive solution of Equation (2) with bounded energy

$$
E(u):=\int_{M}|\nabla u|^{2} d v_{g} \leq \Lambda,
$$

then there exists a positive constant $C$ such that $\|u\|_{C^{3}(M)} \leq C$ and $\min _{M} u \geq C^{-1}$, where $C$ depends on $M, g,\|K\|_{C^{n-2}(M)}, \int_{M} K d v_{g}, \Lambda$, and $\zeta$. 


\section{The example and some notations}

Let $\left(M^{n}, g\right)$ be a compact manifold with $R(g) \equiv 0$ and $n=3$ or 4 . (In fact in this example, $M$ does not need to be locally conformally flat.) We choose $K \in C^{3}(M)$ satisfying the conditions

- $K>0$ somewhere on $M$;

- $\int_{M} K d v_{g} \leq-C_{K}^{-1}<0$ and $\|K\|_{C^{3}(M)} \leq C_{K}$, where $C_{K}$ is a positive constant;

- the set $\{x \in M: K(x)=0\}=\bar{U}$ for some open set $U \subset M$.

We define

$$
K_{i}(x)= \begin{cases}K(x) / i & \text { if } K(x)>0 \\ K(x) & \text { if } K(x) \leq 0\end{cases}
$$

Because on $\partial U$ all derivatives of $K$ up to order 3 are zero, it follows that $K_{i} \in C^{3}(M)$. Furthermore, this definition means that $K_{i} \in \mathscr{K}$, where $\mathscr{K}$ is as defined in Theorem 1.2. Then by Theorem 1.1, there exists a $u_{i}>0$ that satisfies $\Delta_{g} u_{i}+K_{i} u_{i}^{(n+2) /(n-2)}=0$.

Now suppose there is a constant $C$ independent of $i$ such that $\max _{M} u_{i} \leq C$. As proved in [Yan 2007, Section 2], this implies that $\left\{u_{i}\right\}$ is uniformly bounded away from 0 and $\left\|u_{i}\right\|_{C^{3}(M)}$ is bounded above uniformly. Then, passing to a subsequence, $\left\{u_{i}\right\}$ converges in the $C^{2}$ norm to a function $u>0$, and $u$ satisfies $\Delta_{g} u+\tilde{K} u^{(n+2) /(n-2)}=0$, where

$$
\tilde{K}(x)=\lim _{i \rightarrow \infty} K_{i}(x)= \begin{cases}0 & \text { if } K(x)>0, \\ K(x) & \text { if } K(x) \leq 0 .\end{cases}
$$

However, because $\tilde{K}$ is nowhere positive and somewhere negative, the equation $\Delta_{g} u+\tilde{K} u^{(n+2) /(n-2)}=0$ cannot have a positive solution by Theorem 1.1 . This contradiction shows that estimates like the ones in Theorem 1.2 cannot be true without the energy bound assumption on $u$.

Next we prove Theorem 1.4. We first give some definitions and a lemma which will be used in the proof.

Definition 2.1. We call a point $\bar{x}$ on a manifold $M$ a blow-up point of a sequence $\left\{u_{i}\right\}$ if $\bar{x}=\lim _{i \rightarrow \infty} x_{i}$ for some $\left\{x_{i}\right\} \subset M$ and $u_{i}\left(x_{i}\right) \rightarrow \infty$.

Definition 2.2. Suppose $u_{i}$ satisfies $\Delta_{g_{i}} u_{i}-c(n) R\left(g_{i}\right) u_{i}+K_{i} u_{i}^{p_{i}}=0$, where $\left\{g_{i}\right\}$ converges to some metric $g_{0}$. A point $\bar{x} \in M$ is called an isolated blow-up point of $\left\{u_{i}\right\}$ corresponding to $\left\{g_{i}\right\}$ if there exist local maximum points $x_{i}$ of $u_{i}$ and a fixed radius $r_{0}>0$ such that

- $x_{i} \rightarrow \bar{x}$,

- $u_{i}\left(x_{i}\right) \rightarrow \infty$, and 
- $u_{i}(x) \leq C\left(d_{g_{i}}\left(x, x_{i}\right)\right)^{-2 /\left(p_{i}-1\right)}$ for any $x \in B_{r_{0}}\left(x_{i}\right)$, where the constant $C$ is independent of $i$.

Lemma 2.3. If $\bar{x}=\lim _{i \rightarrow \infty} x_{i}$ is an isolated blow-up point of $\left\{u_{i}\right\}$ corresponding to $\left\{g_{i}\right\}$, and $K_{i}$ is uniformly bounded, then there exists a constant $C$ independent of $i$ and $r$ such that, for any $0<r \leq r_{0}$,

$$
\max _{\partial B_{r}\left(x_{i}\right)} u_{i}(x) \leq C \min _{\partial B_{r}\left(x_{i}\right)} u_{i}(x) .
$$

This can be proved as in the proof of [Yan 2007, Lemma 5.2].

Definition 2.4. We say $\bar{x}$ is a simple blow-up point of $\left\{u_{i}\right\}$ if it is an isolated blowup point and there exists an $\bar{r}>0$ independent of $i$ such that $\bar{w}_{i}(r)$ has only one critical point for $r \in(0, \bar{r})$. Here

$$
\bar{w}_{i}(r):=r^{2 /\left(p_{i}-1\right)} \bar{u}_{i}(r)=\operatorname{Vol}\left(S_{r}\right)^{-1} \int_{S_{r}}|z|^{2 /\left(p_{i}-1\right)} u_{i}(z) d \Sigma_{g}
$$

and $z$ is the conformally flat coordinate system centered at each $x_{i}$.

\section{Initial steps of the proof of Theorem 1.4}

The proof of Theorem 1.4 follows along the same line of reasoning as the proof of Theorem 1.2, which is done in [Yan 2007]. As proved in [Section 2] there, a lower bound on $u$ follows directly if there is a uniform upper bound on $u$. By standard elliptic theory and the Sobolev embedding theorem, a bound on the $C^{0}$ norm of $u$ easily implies a bound on its $C^{3}$ norm. Therefore, to prove Theorem 1.4 we only need to show that there is a uniform upper bound on $u$.

By an argument identical to that in [Yan 2007, Section 3], we can show that there exists a positive constant $\eta=\eta\left(M, g, n,\|K\|_{C^{n-2}(M)}, \Lambda\right)$ such that, on the set $K_{\eta}:=\{x \in M: K(x)<\eta\}, u$ has a uniform upper bound depending only on $M, g, n,\|K\|_{C^{n-2}(M)}$, and $\Lambda$. Thus it is left to show that $u$ is uniformly bounded on the set where $K \geq \eta$. We have the following proposition.

Proposition 3.1. Given $\epsilon>0$ and $R \gg 0$, there exists $C=C(\epsilon, R)$ such that, if $u$ is a solution of Equation (2) and

$$
\max _{x \in M}\left(\left(d_{g}\left(x, K_{\eta / 2}\right)\right)^{2 /(p-1)} u(x)\right)>C,
$$

then there exists $\left\{x_{1}, \ldots, x_{N}\right\} \subset M \backslash K_{\eta / 2}$ with $N$ depending on $u$ satifying the following:

- Each $x_{i}$ locally maximizes $u$, and the geodesic balls $\left\{B_{R u\left(x_{i}\right)^{-(p-1) / 2}}\left(x_{i}\right)\right\}$ are disjoint. 
- $|(n+2) /(n-2)-p|<\epsilon$, and, in the coordinate system $y$ chosen so that $z=y / u\left(x_{i}\right)^{(p-1) / 2}$ is the conformally flat coordinate system centered at $x_{i}$, we have

$$
\left\|u\left(x_{i}\right)^{-1} u\left(y / u\left(x_{i}\right)^{(p-1) / 2}\right)-\bar{v}(y)\right\|_{C^{2}\left(B_{2 R}(0)\right)}<\epsilon
$$

on the ball $B_{2 R}(0) \subset \mathbb{R}^{n}(y)$, where

$$
\bar{v}(y)=\left(1+\frac{K\left(x_{i}\right)}{n(n-2)}|y|^{2}\right)^{-(n-2) / 2} .
$$

- There exists $C=C(\epsilon, R)$ such that

$$
u(x) \leq C\left(d_{g}\left(x, \overline{K_{\eta / 2} \bigcup\left\{x_{1}, \ldots, x_{N}\right\}}\right)\right)^{-2 /(p-1)} .
$$

The proof is like that of [Yan 2007, Proposition 4.2], so we omit the details.

Now we are going to prove that $u$ is uniformly bounded on $M \backslash K_{\eta}$. Suppose it is not. Then there are sequences $\left\{u_{i}\right\}$ and $\left\{p_{i}\right\}$ such that

$$
\Delta_{g} u_{i}+K u_{i}^{p_{i}}=0 \quad \text { and } \quad \max _{M \backslash K_{\eta}} u_{i} \rightarrow \infty \text { as } i \rightarrow \infty .
$$

Therefore $\max _{M \backslash K_{\eta}}\left(\left(d_{g}\left(x, K_{\eta / 2}\right)\right)^{2 /\left(p_{i}-1\right)} u_{i}(x)\right) \rightarrow \infty$ as $i \rightarrow \infty$. Then for fixed $\epsilon>0$ and $R \gg 0$ we can apply Proposition 3.1 to each $u_{i}$ and find $x_{1, i}, \ldots, x_{N(i), i}$ such that

- each $x_{j, i}$ for $1 \leq j \leq N(i)$ is a local maximum point of $u_{i}$, and

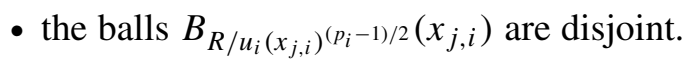

For coordinates $y$ centered at $x_{j, i}$ such that $y / u_{i}\left(x_{j, i}\right)^{\left(p_{i}-1\right) / 2}$ is the conformally flat coordinate system,

$$
\begin{gathered}
\left\|u_{i}\left(x_{j, i}\right)^{-1} u_{i}\left(\frac{y}{u_{i}\left(x_{j, i}\right)^{\left(p_{i}-1\right) / 2}}\right)-\left(1+\frac{K\left(x_{j, i}\right)}{n(n-2)}|y|^{2}\right)^{-(n-2) / 2}\right\|_{C^{2}\left(B_{2 R}(0)\right)}<\epsilon, \\
u_{i}(x) \leq C\left(d_{g}\left(x, \overline{\left.K_{\eta / 2} \bigcup\left\{x_{1, i}, \ldots, x_{N(i), i}\right\}\right)}\right)^{-2 /\left(p_{i}-1\right)}\right.
\end{gathered}
$$

for a constant $C=C(\epsilon, R)$.

Let $\sigma_{i}=\min \left\{d_{g}\left(x_{\alpha, i}, x_{\beta, i}\right): \alpha \neq \beta, 1 \leq \alpha, \beta \leq N(i)\right\}$. Without loss of generality we can assume $\sigma_{i}=d_{g}\left(x_{1, i}, x_{2, i}\right)$. There are two possibilities:

Case I. $\sigma_{i} \geq \varepsilon>0$. In this case, the points $x_{j, i}$ have isolated limiting points $x_{1}, x_{2}, \ldots$, which are isolated blow-up points of $\left\{u_{i}\right\}$ as defined above.

Case II. $\sigma_{i} \rightarrow 0$. In this case, we rescale the coordinates to make the minimal distance 1: let $y=\sigma_{i}^{-1} z$, where $z$ is the conformally flat coordinate system centered at $x_{1, i}$. We also rescale the function by defining $v_{i}(y)=\sigma_{i}^{2 /\left(p_{i}-1\right)} u_{i}\left(\sigma_{i} y\right)$, which satisfies

$$
\Delta_{g^{(i)}} v_{i}+K\left(\sigma_{i} y\right) v_{i}^{p_{i}}=0
$$


where the metric $g^{(i)}(y)=g_{\alpha \beta}\left(\sigma_{i} y\right) d y^{\alpha} d y^{\beta}$. As proved in [Yan 2007, Section 4], 0 is an isolated blow-up point of $\left\{v_{i}\right\}$.

In Sections 4 and 5, we will prove that neither Case I nor Case II can happen.

\section{Ruling out Case I}

If the blow-up points are all isolated, the argument of [Yan 2007, Section 6] shows that among the isolated blow-up points $\left\{x_{1}, x_{2}, \ldots\right\}$, there must be one that is not a simple blow-up point; without loss of generality we assume it to be $x_{1}$. To simplify notation, we are going to rename it $x_{0}$. Let $x_{i}$ be the local maximum point of $u_{i}$ such that $\lim _{i \rightarrow \infty} x_{i}=x_{0}$.

Let $z=\left(z_{1}, \ldots, z_{n}\right)$ be the conformally flat coordinates centered at each $x_{i}$. Since $x_{0}$ is not a simple blow-up point, $|z|^{2 /\left(p_{i}-1\right)} \bar{u}_{i}(|z|)$ has, as a function of $|z|$, a second critical point at $|z|=r_{i}$ where $r_{i} \rightarrow 0$. Let $y=z / r_{i}$ and define $v_{i}(y)=$ $r_{i}^{2 /\left(p_{i}-1\right)} u_{i}\left(r_{i} y\right)$. Then $v_{i}(y)$ satisfies

$$
\Delta_{g^{(i)}} v_{i}(y)+K_{i}(y) v_{i}(y)^{p_{i}}=0,
$$

where $g^{(i)}(y)=g_{\alpha \beta}\left(r_{i} y\right) d y^{\alpha} d y^{\beta}$ and $K_{i}(y)=K\left(r_{i} y\right)$.

By this definition $|y|=1$ is the second critical point of $|y|^{2 /\left(p_{i}-1\right)} \bar{v}_{i}(|y|)$. As shown in [Yan 2007, Section 6], 0 is a simple blow-up point of $\left\{v_{i}\right\}$.

4.1. Estimates for $\boldsymbol{v}_{\boldsymbol{i}}$. The following estimates are essentially the same as [Yan 2007, Proposition 5.3], except for a slightly different choice of parameters. However, we repeat the proof for completeness.

Proposition 4.1. There exists a constant $C$ independent of $i$ such that

- if $0 \leq|y| \leq 1$, then

$$
v_{i}(y) \geq C v_{i}(0)\left(1+\frac{K_{i}(0)}{n(n-2)} v_{i}(0)^{4 /(n-2)}|y|^{2}\right)^{-(n-2) / 2} ;
$$

- if $0 \leq|y| \leq R v_{i}(0)^{-\left(p_{i}-1\right) / 2}$, then

$$
v_{i}(y) \leq C v_{i}(0)\left(1+\frac{K_{i}(0)}{n(n-2)} v_{i}(0)^{p_{i}-1}|y|^{2}\right)^{-(n-2) / 2} ;
$$

- if $R v_{i}(0)^{-\left(p_{i}-1\right) / 2} \leq|y| \leq 1$, then $v_{i}(y) \leq C v_{i}(0)^{t_{i}}|y|^{-l_{i}}$, where $l_{i}$ and $t_{i}$ are chosen so that $(2 n-5) / 2<\lim _{i \rightarrow \infty} l_{i}<n-2$ and $t_{i}=1-\left(p_{i}-1\right) l_{i} / 2$. 
Proof. Let $\rho_{i}:=R v_{i}(0)^{-\left(p_{i}-1\right) / 2}$. By Proposition 3.1, when $0 \leq|y| \leq \rho_{i}$,

$$
\begin{aligned}
(1+\epsilon) v_{i}(0)\left(1+\frac{K_{i}(0)}{n(n-2)} v_{i}(0)^{p_{i}-1}|y|^{2}\right)^{-(n-2) / 2} & \\
& \geq v_{i}(y) \\
& \geq(1-\epsilon) v_{i}(0)\left(1+\frac{K_{i}(0)}{n(n-2)} v_{i}(0)^{p_{i}-1}|y|^{2}\right)^{-(n-2) / 2} \\
& \geq(1-\epsilon) v_{i}(0)\left(1+\frac{K_{i}(0)}{n(n-2)} v_{i}(0)^{4 /(n-2)}|y|^{2}\right)^{-(n-2) / 2} .
\end{aligned}
$$

So we only need to find the upper and lower bounds on $v_{i}(y)$ when $\rho_{i} \leq|y| \leq 1$.

The lower bound. Let $G_{i}$ be the Green's function of $\Delta_{g^{(i)}}$ that is singular at 0 and vanishes on $\partial B_{1}$. Since $\left\{g^{(i)}\right\}$ converges uniformly to the Euclidean metric, there exist constants $C_{1}$ and $C_{2}$ independent of $i$ such that $C_{1}|y|^{2-n} \leq G_{i}(y) \leq C_{2}|y|^{2-n}$.

When $|y|=\rho_{i}$,

$$
\begin{aligned}
v_{i}(y) & \geq(1-\epsilon) v_{i}(0) /\left(1+\frac{K_{i}(0)}{n(n-2)} v_{i}(0)^{p_{i}-1}|y|^{2}\right)^{(n-2) / 2} \\
& =(1-\epsilon) v_{i}(0) /\left(1+\frac{K_{i}(0)}{n(n-2)} R^{2}\right)^{(n-2) / 2} \\
& =(1-\epsilon)\left(R^{-2}+\frac{K_{i}(0)}{n(n-2)}\right)^{-(n-2) / 2} R^{2-n} v_{i}(0) \\
& \geq C R^{2-n} v_{i}(0) \\
& \geq C R^{2-n} v_{i}(0)^{(n-2)\left(p_{i}-1\right) / 2-1} \quad\left(\text { since }(n-2)\left(p_{i}-1\right) / 2-1 \leq 1\right) \\
& =C v_{i}(0)^{-1}|y|^{2-n} \\
& \geq C v_{i}(0)^{-1} G_{i}(y) .
\end{aligned}
$$

With this constant $C$, we have $C v_{i}(0)^{-1} G_{i}(y)=0<v_{i}(y)$ when $|y|=1$,

We know that

$$
\Delta_{g^{(i)}}\left(v_{i}(y)-C v_{i}(0)^{-1} G_{i}(y)\right)=\Delta_{g^{(i)}} v_{i}(y)=-K_{i}(y) v_{i}(y)^{p_{i}}<0
$$

on $B_{1} \backslash B_{\rho_{i}}$. Therefore, by the maximal principle, when $\rho_{i} \leq|y| \leq 1$,

$$
v_{i}(y)>C v_{i}(0)^{-1} G_{i}(y) \geq C v_{i}(0)^{-1}|y|^{2-n} .
$$

Now we need to compare $|y|^{2-n} v_{i}(0)^{-1}$ with

$$
v_{i}(0) \cdot\left(1+\frac{K_{i}(0)}{n(n-2)} v_{i}(0)^{4 /(n-2)}|y|^{2}\right)^{-(n-2) / 2}
$$


in order to get the desired lower bound:

$$
\begin{aligned}
v_{i}(0)^{2}|y|^{n-2}\left(1+\frac{K_{i}(0)}{n(n-2)} v_{i}(0)^{4 /(n-2)}|y|^{2}\right)^{-(n-2) / 2} & \\
& \leq v_{i}(0)^{2}\left(\frac{K_{i}(0)}{n(n-2)} v_{i}(0)^{4 /(n-2)}\right)^{-(n-2) / 2} \leq C
\end{aligned}
$$

for a constant $C$ independent of $i$. Therefore

$$
v_{i}(0)^{-1}|y|^{2-n} \geq C v_{i}(0)\left(1+\frac{K_{i}(0)}{n(n-2)} v_{i}(0)^{4 /(n-2)}|y|^{2}\right)^{-(n-2) / 2},
$$

and consequently

$$
v_{i}(y) \geq C v_{i}(0)\left(1+\frac{K_{i}(0)}{n(n-2)} v_{i}(0)^{4 /(n-2)}|y|^{2}\right)^{-(n-2) / 2}
$$

when $\rho_{i} \leq|y| \leq 1$.

The upper bound. We are going to apply the same strategy of constructing a comparison function and using the maximal principle.

Define $\mathscr{L}_{i} \varphi:=\Delta_{g^{(i)}} \varphi+K_{i} v_{i}^{p_{i}-1} \varphi$. By this definition $\mathscr{L}_{i} v_{i}=0$. Let $M_{i}=$ $\max _{\partial B_{1}} v_{i}$ and $C_{i}=(1+\epsilon)\left(K_{i}(0) /(n(n-2))\right)^{-(n-2) / 2}$. Note that $C_{i}$ is bounded above and below by constants independent of $i$. Consider the function

$$
M_{i}|y|^{-n+2+l_{i}}+C_{i} v_{i}(0)^{t_{i}}|y|^{-l_{i}} .
$$

When $|y|=\rho_{i}$,

$$
\begin{aligned}
v_{i}(y) & \leq(1+\epsilon) v_{i}(0) /\left(1+\frac{K_{i}(0)}{n(n-2)} v_{i}(0)^{p_{i}-1}|y|^{2}\right)^{(n-2) / 2} \\
& =(1+\epsilon) v_{i}(0) /\left(1+\frac{K_{i}(0)}{n(n-2)} R^{2}\right)^{(n-2) / 2} \\
& \leq C_{i} v_{i}(0) R^{-(n-2)} \\
& \leq C_{i} v_{i}(0) R^{-l_{i}} \\
& =C_{i} v_{i}(0)^{t_{i}}|y|^{-l_{i}} .
\end{aligned}
$$

When $|y|=1$, the definition of $M_{i}$ gives $v_{i}(y) \leq M_{i}=M_{i}|y|^{-n+2+l_{i}}$. Thus on $\{|y|=1\} \bigcup\left\{|y|=\rho_{i}\right\}$, we have $v_{i}(y) \leq M_{i}|y|^{-n+2+l_{i}}+C_{i} v_{i}(0)^{t_{i}}|y|^{-l_{i}}$.

In Euclidean coordinates,

$$
\Delta|y|^{-l_{i}}=-l_{i}\left(n-2-l_{i}\right)|y|^{-l_{i}-2} \text { and } \Delta|y|^{-n+2+l_{i}}=-l_{i}\left(n-2-l_{i}\right)|y|^{-n+l_{i}} .
$$

When $i$ is sufficiently large, $g^{(i)}$ is close to the Euclidean metric. Therefore

$$
\Delta_{g^{(i)}}|y|^{-l_{i}} \leq-\frac{1}{2} l_{i}\left(n-2-l_{i}\right)|y|^{-l_{i}-2}
$$


and

$$
\Delta_{g^{(i)}}|y|^{-n+2+l_{i}} \leq-\frac{1}{2} l_{i}\left(n-2-l_{i}\right)|y|^{-n+l_{i}} .
$$

Thus

$$
\begin{aligned}
\mathscr{L}_{i}\left(C_{i} v_{i}(0)^{t_{i}}|y|^{-l_{i}}\right) & =C_{i} v_{i}(0)^{t_{i}} \Delta_{g^{(i)}}|y|^{-l_{i}}+C_{i} v_{i}(0)^{t_{i}} K_{i} v_{i}(y)^{p_{i}-1}|y|^{-l_{i}} \\
& \leq-C l_{i}\left(n-2-l_{i}\right) v_{i}(0)^{t_{i}}|y|^{-l_{i}-2}+C^{\prime} v_{i}(0)^{t_{i}} v_{i}(y)^{p_{i}-1}|y|^{-l_{i}}
\end{aligned}
$$

for some constants $C$ and $C^{\prime}$ independent of $i$.

Lemma 2.3 and the upper bound on $v_{i}(y)$ when $|y| \leq \rho_{i}$ imply that

$$
\bar{v}_{i}\left(\rho_{i}\right) \leq C \frac{(1+\epsilon) v_{i}(0)}{\left(1+\frac{K_{i}(0)}{n(n-2)} v_{i}(0)^{p_{i}-1}\left(\rho_{i}\right)^{2}\right)^{(n-2) / 2}} \leq C v_{i}(0) R^{2-n} .
$$

Then since 0 is a simple blow-up point and $r^{2 /\left(p_{i}-1\right)} \bar{v}_{i}(r)$ is decreasing from $\rho_{i}$ to 1 , we have

$$
\begin{aligned}
|y|^{2 /\left(p_{i}-1\right)} \bar{v}_{i}(|y|) & \leq \rho_{i}{ }^{2 /\left(p_{i}-1\right)} \cdot \bar{v}_{i}\left(\rho_{i}\right) \\
& \leq C R^{2 /\left(p_{i}-1\right)+2-n} .
\end{aligned}
$$

Thus again by Lemma 2.3, we have

$$
v_{i}(y)^{p_{i}-1} \leq C \bar{v}_{i}(|y|)^{p_{i}-1} \leq C|y|^{-2} R^{2-(n-2)\left(p_{i}-1\right)},
$$

and hence $v_{i}(y)^{p_{i}-1}|y|^{-l_{i}} \leq C|y|^{-2-l_{i}} R^{2-(n-2)\left(p_{i}-1\right)}$. Therefore

$$
\mathscr{L}_{i}\left(C_{i} v_{i}(0)^{t_{i}}|y|^{-l_{i}}\right) \leq\left(-C l_{i}\left(n-2-l_{i}\right)+C^{\prime} R^{2-(n-2)\left(p_{i}-1\right)}\right) v_{i}(0)^{t_{i}}|y|^{-l_{i}-2} .
$$

Our choice of $l_{i}$ means that $l_{i}\left(n-2-l_{i}\right)$ is always bounded below by some positive constant independent of $i$. When $i$ is sufficiently large, $2-(n-2)\left(p_{i}-1\right)<0$, so we can choose $R$ big enough so that $-C l_{i}\left(n-2-l_{i}\right)+C^{\prime} R^{2-(n-2)\left(p_{i}-1\right)}<0$, which implies $\mathscr{L}_{i}\left(C_{i} v_{i}(0)^{t_{i}}|y|^{-l_{i}}\right)<0$.

Similarly,

$$
\begin{aligned}
\mathscr{L}_{i}\left(M_{i}|y|^{-n+2+l_{i}}\right) & =M_{i} \Delta_{g^{(i)}}|y|^{-n+2+l_{i}}+M_{i} K_{i} v_{i}^{p_{i}-1}|y|^{-n+2+l_{i}} \\
& \leq-\frac{1}{2} l_{i}\left(n-2-l_{i}\right) M_{i}|y|^{-n+l_{i}}+K_{i} M_{i} R^{2-(n-2)\left(p_{i}-1\right)}|y|^{-n+l_{i}}
\end{aligned}
$$

by Equations (3) and (4). We can choose $R$ large enough so that

$$
-\frac{1}{2} l_{i}\left(n-2-l_{i}\right)+K_{i} R^{2-(n-2)\left(p_{i}-1\right)}<0,
$$

and hence $\mathscr{L}_{i}\left(M_{i}|y|^{-n+2+l_{i}}\right)<0$. Therefore, when $\rho_{i} \leq|y| \leq 1$,

$$
\mathscr{L}_{i}\left(M_{i}|y|^{-n+2+l_{i}}+C_{i} v_{i}(0)^{t_{i}}|y|^{-l_{i}}\right)<0 .
$$

Then, by the maximal principle, $v_{i}(y) \leq M_{i}|y|^{-n+2+l_{i}}+C_{i} v_{i}(0)^{t_{i}}|y|^{-l_{i}}$.

By Lemma 2.3 and because 0 is a simple blow-up point, we have 


$$
\begin{aligned}
M_{i} \leq C \theta^{2 /\left(p_{i}-1\right)} \bar{v}_{i}(\theta) & \leq C \theta^{2 /\left(p_{i}-1\right)}\left(M_{i} \theta^{-n+2+l_{i}}+C_{i} v_{i}(0)^{t_{i}} \theta^{-l_{i}}\right) \\
& =C \theta^{2 /\left(p_{i}-1\right)-n+2+l_{i}} M_{i}+C \theta^{2 /\left(p_{i}-1\right)} \cdot C_{i} v_{i}(0)^{t_{i}} \theta^{-l_{i}}
\end{aligned}
$$

for $\rho_{i} \leq \theta \leq 1$ and some constant $C$ independent of $i$.

Note that

$$
\lim _{i \rightarrow \infty}\left(\frac{2}{p_{i}-1}-n+2+l_{i}\right)=-\frac{n-2}{2}+\lim _{i \rightarrow \infty} l_{i}>-\frac{n-2}{2}+\frac{2 n-5}{2}>0
$$

because $n \geq 5$.

Since $\rho_{i} \rightarrow 0$, we can choose $\theta$ small enough (fixed and independent of $i$ ) to absorb the first term on the right side of the above inequality into the left side. We then get $M_{i} \leq 2 C \theta^{2 /\left(p_{i}-1\right)} \cdot C_{i} v_{i}(0)^{t_{i}} \theta^{-l_{i}} \leq C v_{i}(0)^{t_{i}}$.

Therefore

$$
\begin{aligned}
v_{i}(y) & \leq M_{i}|y|^{-n+2+l_{i}}+C_{i} v_{i}(0)^{t_{i}}|y|^{-l_{i}} \\
& \leq M_{i}|y|^{-l_{i}}+C_{i} v_{i}(0)^{t_{i}}|y|^{-l_{i}} \leq C v_{i}(0)^{t_{i}}|y|^{-l_{i}} .
\end{aligned}
$$

4.2. A preliminary estimate for $\delta_{i}:=(n+2) /(n-2)-p_{i}$. First we prove a technical lemma.

Lemma 4.2. When $\sigma<1$ and $0 \leq \kappa \leq n-2$,

$$
\int_{|y| \leq \sigma}|y|^{\kappa} v_{i}(y)^{p_{i}+1} d y \leq C v_{i}(0)^{-2 \kappa /(n-2)+(n-2+\kappa) / 2 \delta_{i}},
$$

where $C$ is independent of $i$.

Proof. Let $\rho_{i}:=R v_{i}(0)^{-\left(p_{i}-1\right) / 2}$. By Proposition 4.1

$$
\begin{aligned}
\int_{|y| \leq \rho_{i}}|y|^{\kappa} v_{i}(y)^{p_{i}+1} d y & \leq C v_{i}(0)^{p_{i}+1} \int_{|y| \leq \rho_{i}}|y|^{\kappa} d y \\
& \leq C v_{i}(0)^{p_{i}+1-(n+\kappa)\left(p_{i}-1\right) / 2}=C v_{i}(0)^{-2 \kappa /(n-2)+(n-2+\kappa) / 2 \delta_{i}} .
\end{aligned}
$$

Since $n \geq 5$, our choice of $l_{i}$ gives

$$
\begin{aligned}
\lim _{i \rightarrow \infty}\left(n+\kappa-l_{i}\left(p_{i}+1\right)\right) & =n+\kappa-\frac{2 n}{n-2} \lim _{i \rightarrow \infty} l_{i} \\
& <n+\kappa-\frac{2 n}{n-2} \cdot \frac{2 n-5}{2} \leq n+(n-2)-\frac{n(2 n-5)}{n-2}<0 .
\end{aligned}
$$

Therefore

$$
\begin{aligned}
\int_{\rho_{i} \leq|y| \leq \sigma}|y|^{\kappa} v_{i}(y)^{p_{i}+1} d y & \leq C \int_{\rho_{i} \leq|y| \leq \sigma}|y|^{\kappa}\left(v_{i}(0)^{t_{i}}|y|^{-l_{i}}\right)^{p_{i}+1} d y \\
& \leq C v_{i}(0)^{t_{i}\left(p_{i}+1\right)-\left(p_{i}-1\right)\left(n-l_{i}\left(p_{i}+1\right)+\kappa\right) / 2} \\
& =C v_{i}(0)^{p_{i}+1-(n+\kappa)\left(p_{i}-1\right) / 2} \quad\left(\text { by the definition of } t_{i}\right) \\
& =C v_{i}(0)^{-2 \kappa /(n-2)+(n-2+\kappa) \delta_{i} / 2} .
\end{aligned}
$$


Thus

$$
\int_{|y| \leq \sigma}|y|^{\kappa} v_{i}(y)^{p_{i}+1} d y \leq C v_{i}(0)^{-2 \kappa /(n-2)+(n-2+\kappa) \delta_{i} / 2} .
$$

The next proposition is a preliminary estimate for $\delta_{i}:=(n+2) /(n-2)-p_{i}$. We will also derive a refined estimate in a later part of this paper.

Proposition 4.3. $\lim _{i \rightarrow \infty} v_{i}(0)^{\delta_{i}}=1$.

Proof. Since the original metric is locally conformally flat, it can be written locally as $\lambda(z)^{4 /(n-2)} d z^{2}$. Let $\lambda_{i}(y)=\lambda\left(r_{i} y\right)$. Then $g^{(i)}(y)=\lambda_{i}(y)^{4 /(n-2)} d y^{2}$. Let $\sigma<1$. The Pohozaev identity in [Schoen 1988b] says that, for a conformal Killing field $X$ on $B_{\sigma}$,

$$
\frac{n-2}{2 n} \int_{B_{\sigma}} X\left(R_{i}\right) d v_{g_{i}}=\int_{\partial B_{\sigma}} T_{i}\left(X, v_{i}\right) d \Sigma_{i}
$$

where the notations are as follows:

$$
\begin{array}{rlrl}
g_{i} & =v_{i}^{4 /(n-2)} g^{(i)}=\left(\lambda_{i} v_{i}\right)^{4 /(n-2)} d y^{2} ; & & d v_{g_{i}}=\left(\lambda_{i} v_{i}\right)^{2 n /(n-2)} d y ; \\
R_{i} & =R\left(g_{i}\right)=c(n)^{-1} K_{i} v_{i}^{-\delta_{i}} ; & \\
v_{i}=\left(\lambda_{i} v_{i}\right)^{-2 /(n-2)} \sigma^{-1} \sum_{j} y^{j} \partial / \partial y^{j} & & \text { is the unit outer normal vector on } \partial B_{\sigma} \\
d \Sigma_{i} & =\left(\lambda_{i} v_{i}\right)^{2(n-1) /(n-2)} d \Sigma_{\sigma}, & & \text { with respect to } g_{i} ; \\
T_{i} & =\operatorname{Ric}\left(g_{i}\right)-n^{-1} R\left(g_{i}\right) g_{i} & & \text { where } d \Sigma_{\sigma} \text { is the surface element of } \\
& & \text { is the traceless Ricci tensor with re- } & \text { spect to } g_{i} .
\end{array}
$$

According to [Schoen 1989], $T_{i}$ can also be expressed as

$$
(n-2)\left(\lambda_{i} v_{i}\right)^{2 /(n-2)}\left(\operatorname{Hess}\left(\left(\lambda_{i} v_{i}\right)^{-2 /(n-2)}\right)-\frac{1}{n} \Delta\left(\left(\lambda_{i} v_{i}\right)^{-2 /(n-2)}\right) d y^{2}\right),
$$

where Hess and $\Delta$ are taken with respect to the Euclidean metric $d y^{2}$.

We choose $X=\sum_{j=1}^{n} y^{j} \partial / \partial y^{j}$. Up to the constant $2(n-1) / n$, the integral in the left side of (5) is equal to

$$
\begin{aligned}
\int_{B_{\sigma}} X\left(K_{i} v_{i}^{-\delta_{i}}\right)\left(\lambda_{i}\right. & \left.v_{i}\right)^{2 n /(n-2)} d y \\
& =\int_{B_{\sigma}} X\left(K_{i}\right) v_{i}^{p_{i}+1} \lambda_{i}^{2 n /(n-2)} d y-\delta_{i} \int_{B_{\sigma}} K_{i} v_{i}^{p_{i}} X\left(v_{i}\right) \lambda_{i}^{2 n /(n-2)} d y .
\end{aligned}
$$


By the divergence theorem, this is equal to

$$
\begin{aligned}
& \int_{B_{\sigma}}|y| \frac{\partial K_{i}}{\partial r} v_{i}^{p_{i}+1} \lambda_{i}^{2 n /(n-2)} d y \\
& +\frac{\delta_{i}}{p_{i}+1}\left(\int_{B_{\sigma}} r \frac{\partial K_{i}}{\partial r} \lambda_{i}^{2 n /(n-2)} v_{i}^{p_{i}+1} d y+\int_{B_{\sigma}} K_{i} v_{i}^{p_{i}+1} r \frac{\partial \lambda_{i}^{2 n /(n-2)}}{\partial r} d y\right. \\
& \left.\quad+\int_{B_{\sigma}} K_{i} v_{i}^{p_{i}+1} \lambda_{i}^{2 n /(n-2)} \operatorname{div} X d y\right) \\
& -\frac{\delta_{i}}{p_{i}+1} \int_{\partial B_{\sigma}} K_{i} v_{i}^{p_{i}+1} \lambda_{i}^{2 n /(n-2)} X \cdot\left(\left(\sum y^{j} \partial / \partial y^{j}\right) / \sigma\right) d \Sigma_{\sigma} .
\end{aligned}
$$

Restoring the factor $2(n-1) / n$, we can now write the left side of (5) as

$$
\begin{gathered}
\text { (6) } \frac{2(n-1)}{n}\left(1+\frac{\delta_{i}}{p_{i}+1}\right) \int_{B_{\sigma}}|y| \frac{\partial K_{i}}{\partial r} v_{i}^{p_{i}+1} \lambda_{i}^{2 n /(n-2)} d y \\
+\frac{2(n-1)}{n} \frac{\delta_{i}}{p_{i}+1} \int_{B_{\sigma}}|y| K_{i} v_{i}^{p_{i}+1} \frac{\partial \lambda_{i}^{2 n /(n-2)}}{\partial r} d y+\frac{2(n-1)}{n} \frac{\delta_{i} n}{p_{i}+1} \int_{B_{\sigma}} K_{i} v_{i}^{p_{i}+1} \lambda_{i}^{2 n /(n-2)} d y \\
\quad-\frac{2(n-1)}{n} \frac{\delta_{i}}{p_{i}+1} \int_{\partial B_{\sigma}} \sigma K_{i} v_{i}^{p_{i}+1} \lambda_{i}^{2 n /(n-2)} d \Sigma_{\sigma} .
\end{gathered}
$$

The right side of (5) is

$$
\begin{gathered}
\int_{\partial B_{\sigma}}(n-2)\left(\lambda_{i} v_{i}\right)^{2 /(n-2)}\left(\operatorname{Hess}\left(\left(\lambda_{i} v_{i}\right)^{-2 /(n-2)}\right)\left(r \frac{\partial}{\partial r},\left(\lambda_{i} v_{i}\right)^{-2 /(n-2)} \sigma^{-1} r \frac{\partial}{\partial r}\right)\right. \\
\left.-\frac{1}{n} \Delta\left(\left(\lambda_{i} v_{i}\right)^{-2 /(n-2)}\right)\left\langle r \frac{\partial}{\partial r},\left(\lambda_{i} v_{i}\right)^{-2 /(n-2)} \sigma^{-1} r \frac{\partial}{\partial r}\right)\right)\left(\lambda_{i} v_{i}\right)^{2(n-1) /(n-2)} d \Sigma_{\sigma} \\
=(n-2) \int_{\partial B_{\sigma}}\left(\sigma^{-1} \operatorname{Hess}\left(\left(\lambda_{i} v_{i}\right)^{-2 /(n-2)}\right)\left(r \frac{\partial}{\partial r}, r \frac{\partial}{\partial r}\right)\right. \\
\left.-\frac{\sigma}{n} \Delta\left(\left(\lambda_{i} v_{i}\right)^{-2 /(n-2)}\right)\right)\left(\lambda_{i} v_{i}\right)^{2(n-1) /(n-2)} d \Sigma_{\sigma} \\
\text { (7) }=(n-2) \int_{\partial B_{\sigma}}\left(\sigma ^ { - 1 } \left(-\frac{2}{n-2}\left(\lambda_{i} v_{i}\right) \sum_{j, k} y^{j} y^{k} \frac{\partial}{\partial y^{k}} \frac{\partial}{\partial y^{j}}\left(\lambda_{i} v_{i}\right)\right.\right. \\
\left.\quad+\frac{2 n}{(n-2)^{2}} \sum_{j, k} y^{j} y^{k} \frac{\partial\left(\lambda_{i} v_{i}\right)}{\partial y^{k}} \frac{\partial\left(\lambda_{i} v_{i}\right)}{\partial y^{j}}\right) \\
\left.-\sigma \cdot\left(-\frac{2}{n(n-2)}\left(\lambda_{i} v_{i}\right) \sum_{j} \frac{\partial^{2}\left(\lambda_{i} v_{i}\right)}{\left(\partial y^{j}\right)^{2}}+\frac{2}{(n-2)^{2}} \sum_{j}\left(\frac{\partial\left(\lambda_{i} v_{i}\right)}{\partial y^{j}}\right)^{2}\right)\right) d \Sigma_{\sigma} .
\end{gathered}
$$

Next we are going to study the decay rate of each term in (6) and (7). 
On $\partial B_{\sigma}$, Proposition 4.1 implies $v_{i} \leq C v_{i}(0)^{t_{i}}$. Then by the elliptic regularity theory [Gilbarg and Trudinger 2001], we have $\left\|v_{i}\right\|_{C^{2}\left(\partial B_{\sigma}\right)} \leq C v_{i}(0)^{t_{i}}$. Thus we know (7) decays at a rate of $v_{i}(0)^{2 t_{i}}$.

The fourth term in (6) decays in the order of $\delta_{i} v_{i}(0)^{t_{i}\left(p_{i}+1\right)}$ by Proposition 4.1. By Lemma 4.2, we know that the second term in (6) is bounded above by

$$
C \delta_{i} \int_{B_{\sigma}}|y| v_{i}^{p_{i}+1} d y \leq C \delta_{i} v_{i}(0)^{-2 /(n-2)+(n-1) \delta_{i} / 2} .
$$

Therefore the sum of the first and the third terms in (6), which is

$$
\begin{aligned}
\frac{n}{2(n-1)}\left(1+\frac{\delta_{i}}{p_{i}+1}\right) \int_{B_{\sigma}}|y| \frac{\partial K_{i}}{\partial r} v_{i}^{p_{i}+1} \lambda_{i}^{2 n /(n-2)} d y & \\
& +\frac{n}{2(n-1)} \frac{\delta_{i}}{p_{i}+1} n \int_{B_{\sigma}} K_{i} v_{i}^{p_{i}+1} \lambda_{i}^{2 n /(n-2)} d y,
\end{aligned}
$$

is bounded above by $C v_{i}(0)^{2 t_{i}}+C \delta_{i} v_{i}(0)^{t_{i}\left(p_{i}+1\right)}+C \delta_{i} v_{i}(0)^{-2 /(n-2)+(n-1) \delta_{i} / 2}$.

By our choice of $l_{i}$ and $t_{i}$, we have, as $i \rightarrow \infty$,

$$
t_{i}=1-\frac{\left(p_{i}-1\right) l_{i}}{2} \rightarrow 1-\frac{2}{n-2} \lim _{i \rightarrow \infty} l_{i}<1-\frac{2}{n-2} \cdot \frac{2 n-5}{2}<0 .
$$

Thus $C v_{i}(0)^{2 t_{i}}+C \delta_{i} v_{i}(0)^{t_{i}\left(p_{i}+1\right)} \leq C v_{i}(0)^{2 t_{i}}+C v_{i}(0)^{t_{i}\left(p_{i}+1\right)} \leq C v_{i}(0)^{2 t_{i}}$.

On the other hand,

$$
\frac{\delta_{i}}{p_{i}+1} n \int_{B_{\sigma}} K_{i} v_{i}^{p_{i}+1} \lambda_{i}^{2 n /(n-2)} d y \geq C \delta_{i} \int_{B_{\sigma}} v_{i}^{p_{i}+1} d y .
$$

When $|y| \leq \rho_{i}$, Proposition 4.1 gives

$$
\begin{aligned}
v_{i}(y) & \geq(1-\epsilon) v_{i}(0) /\left(1+\frac{K_{i}(0)}{n(n-2)} v_{i}(0)^{p_{i}-1}|y|^{2}\right)^{(n-2) / 2} \\
& \geq(1-\epsilon) v_{i}(0) /\left(1+\frac{K_{i}(0)}{n(n-2)} R^{2}\right)^{(n-2) / 2} \geq C v_{i}(0),
\end{aligned}
$$

so

$$
\begin{aligned}
\int_{B_{\sigma}} v_{i}^{p_{i}+1} d y>\int_{|y| \leq \rho_{i}} v_{i}^{p_{i}+1} d y \geq C v_{i}(0)^{p_{i}+1-n\left(p_{i}-1\right) / 2} & =C v_{i}(0)^{(n-2) \delta_{i} / 2} \\
& \geq C .
\end{aligned}
$$

This implies that the third term in (6) is bounded below by $C \delta_{i}$.

Then by comparing the decay rates of the terms in (6) and (7),

$$
\delta_{i} \leq C\left(v_{i}(0)^{2 t_{i}}+\delta_{i} v_{i}(0)^{-2 /(n-2)+(n-1) \delta_{i} / 2}+\left|\int_{B_{\sigma}} \frac{\partial K_{i}}{\partial r}\right| y\left|v_{i}^{p_{i}+1} \lambda_{i}^{2 n /(n-2)} d y\right|\right) .
$$


Since $v_{i}(0)^{-2 /(n-2)+(n-1) \delta_{i} / 2} \rightarrow 0$, the second term on the right side can be absorbed into the left side. Thus we conclude that

$$
\delta_{i} \leq C\left(v_{i}(0)^{2 t_{i}}+\left|\int_{B_{\sigma}} \frac{\partial K_{i}}{\partial r}\right| y\left|v_{i}^{p_{i}+1} \lambda_{i}^{2 n /(n-2)} d y\right|\right) .
$$

By Lemma 4.2,

$$
\left|\int_{B_{\sigma}} \frac{\partial K_{i}}{\partial r}\right| y\left|v_{i}^{p_{i}+1} \lambda_{i}^{2 n /(n-2)} d y\right| \leq C v_{i}(0)^{-2 /(n-2)+(n-1) \delta_{i} / 2} .
$$

Thus $\delta_{i} \leq C\left(v_{i}(0)^{-2 /(n-2)+(n-1) \delta_{i} / 2}+v_{i}(0)^{2 t_{i}}\right)$. This implies that

$$
\delta_{i} \ln v_{i}(0) \leq C\left(v_{i}(0)^{-2 /(n-2)+(n-1) \delta_{i} / 2}+v_{i}(0)^{2 t_{i}}\right) \ln v_{i}(0) \rightarrow 0
$$

as $i \rightarrow \infty$. Therefore $\lim _{i \rightarrow \infty} v_{i}(0)^{\delta_{i}}=1$. Consequently, we have

$$
\delta_{i} \leq C\left(v_{i}(0)^{-2 /(n-2)}+v_{i}(0)^{2 t_{i}}\right) .
$$

4.3. A preliminary estimate for $\left|\nabla K_{i}\right|$. We will again study the Pohozaev identity (5), but with a different choice of the conformal Killing field $X=\partial / \partial y^{1}$.

Direct calculation, like that in the proof of Proposition 4.3, shows that the right side of the identity is equal to

$$
\begin{aligned}
(n-2) \int_{\partial B_{\sigma}}( & \sum_{j} \frac{y^{j}}{\sigma}\left(-\frac{2}{n-2}\left(\lambda_{i} v_{i}\right) \frac{\partial^{2}\left(\lambda_{i} v_{i}\right)}{\partial y^{1} \partial y^{j}}+\frac{2 n}{(n-2)^{2}} \frac{\partial\left(\lambda_{i} v_{i}\right)}{\partial y^{1}} \frac{\partial\left(\lambda_{i} v_{i}\right)}{\partial y^{j}}\right) \\
& \left.-\frac{y^{1}}{\sigma} \sum_{j}\left(-\frac{2}{n(n-2)}\left(\lambda_{i} v_{i}\right) \frac{\partial^{2}\left(\lambda_{i} v_{i}\right)}{\left(\partial y^{j}\right)^{2}}+\frac{2}{(n-2)^{2}}\left(\frac{\partial\left(\lambda_{i} v_{i}\right)}{\partial y^{j}}\right)^{2}\right)\right) d \Sigma_{\sigma},
\end{aligned}
$$

and decays at a rate of $v_{i}(0)^{2 t_{i}}$.

The left side of this identity is $(n-2) /(2 n)$ times

$$
\begin{aligned}
\int_{B_{\sigma}} \frac{\partial}{\partial y^{1}}\left(R_{i}\right) d v_{g_{i}}= & c(n)^{-1} \int_{B_{\sigma}} \frac{\partial}{\partial y^{1}}\left(K_{i} v_{i}^{-\delta_{i}}\right)\left(\lambda_{i} v_{i}\right)^{2 n /(n-2)} d y \\
= & c(n)^{-1} \int_{B_{\sigma}}\left(1+\frac{\delta_{i}}{p_{i}+1}\right) \lambda_{i}^{2 n /(n-2)} v_{i}^{p_{i}+1} \frac{\partial K_{i}}{\partial y^{1}} d y \\
& +c(n)^{-1} \int_{B_{\sigma}} \frac{\delta_{i}}{p_{i}+1} K_{i} v_{i}^{p_{i}+1} \frac{\partial \lambda_{i}^{2 n /(n-2)}}{\partial y^{1}} d y \\
& -c(n)^{-1} \frac{\delta_{i}}{p_{i}+1} \int_{\partial B_{\sigma}} \lambda_{i}^{2 n /(n-2)} K_{i} v_{i}^{p_{i}+1} \frac{y^{1}}{\sigma} d \Sigma_{\sigma} .
\end{aligned}
$$

By Proposition 4.1, the last term in (11) is bounded from above by

$$
C \delta_{i} \cdot v_{i}(0)^{t_{i}\left(p_{i}+1\right)} \leq C \delta_{i} v_{i}(0)^{2 t_{i}},
$$


since $t_{i}<0$ and $v_{i}(0) \rightarrow \infty$.

Note that since $\lambda_{i}(y)=\lambda\left(r_{i} y\right)$, the second term in (11) is bounded from above by

$$
C \delta_{i} r_{i} \int_{|y| \leq \sigma} v_{i}(y)^{p_{i}+1} d y,
$$

which is further bounded by $C \delta_{i} r_{i} v_{i}(0)^{(n-2) / 2 \delta_{i}} \leq C \delta_{i} r_{i}$ by Lemma 4.2 and Proposition 4.3.

Therefore the first term in (11), which is

$$
c(n)^{-1} \int_{B_{\sigma}}\left(1+\frac{\delta_{i}}{p_{i}+1}\right) \lambda_{i}^{2 n /(n-2)} v_{i}^{p_{i}+1} \frac{\partial K_{i}}{\partial y^{1}} d y,
$$

is bounded from above by $C\left(v_{i}(0)^{2 t_{i}}+\delta_{i} v_{i}(0)^{2 t_{i}}+\delta_{i} r_{i}\right) \leq C\left(\delta_{i} r_{i}+v_{i}(0)^{2 t_{i}}\right)$.

This shows that

$$
\left|\int_{B_{\sigma}} \lambda_{i}^{2 n /(n-2)} v_{i}^{p_{i}+1} \frac{\partial K_{i}}{\partial y^{1}} d y\right| \leq C\left(\delta_{i} r_{i}+v_{i}(0)^{2 t_{i}}\right) .
$$

By the Taylor expansion,

$$
\frac{\partial K_{i}}{\partial y^{1}}(y)=\frac{\partial K_{i}}{\partial y^{1}}(0)+\nabla\left(\frac{\partial K_{i}}{\partial y^{1}}\right)(\varsigma) \cdot y \quad \text { for some }|\varsigma| \leq|y| .
$$

Note that $K_{i}(y)=K\left(r_{i} y\right)$. By Lemma 4.2 and Proposition 4.3,

$$
\begin{aligned}
\int_{B_{\sigma}} \lambda_{i}^{2 n /(n-2)} v_{i}^{p_{i}+1}\left|\nabla\left(\frac{\partial K_{i}}{\partial y^{1}}\right)(\varsigma) \cdot y\right| d y & \leq C r_{i} \int_{B_{\sigma}} v_{i}^{p_{i}+1}|y| d y \\
& \leq C r_{i} v_{i}(0)^{-2 /(n-2)+(n-1) \delta_{i} / 2} \\
& \leq C r_{i} v_{i}(0)^{-2 /(n-2)} .
\end{aligned}
$$

Thus we know

$$
\begin{aligned}
\left|\frac{\partial K_{i}}{\partial y^{1}}(0)\right| \int_{B_{\sigma}} v_{i}^{p_{i}+1} d y & \leq C\left|\int_{B_{\sigma}} \lambda_{i}^{2 n /(n-2)} v_{i}^{p_{i}+1} \frac{\partial K_{i}}{\partial y^{1}}(0) d y\right| \\
& \leq C\left(r_{i} v_{i}(0)^{-2 /(n-2)}+\left(\delta_{i} r_{i}+v_{i}(0)^{2 t_{i}}\right)\right) \\
& \leq C\left(r_{i} v_{i}(0)^{-2 /(n-2)}+r_{i} v_{i}(0)^{2 t_{i}}+v_{i}(0)^{2 t_{i}}\right) \\
& \leq C\left(r_{i} v_{i}(0)^{-2 /(n-2)}+v_{i}(0)^{2 t_{i}}\right) .
\end{aligned}
$$

Then by (8),

$$
\left|\frac{\partial K_{i}}{\partial y^{1}}(0)\right| \leq C\left(r_{i} v_{i}(0)^{-2 /(n-2)}+v_{i}(0)^{2 t_{i}}\right) .
$$

The same estimate holds for $\left|\partial K_{i} / \partial y^{j}(0)\right|$ for $j=2, \ldots, n$ as well, since we can also choose $X=\partial / \partial y^{j}$ in the above calculation. 
4.4. Location of the blow-up. Choose a point $\bar{y}$ with $|\bar{y}|=1$. It is proved in [Yan 2007, Section 6] that $v_{i} / v_{i}(\bar{y})$ converges in $C^{2}$ norm to a function $h$ on any compact subset of $\mathbb{R}^{n} \backslash\{0\}$, and $h=\frac{1}{2}+\frac{1}{2}|y|^{2-n}$.

Recall that we chose the coordinate systems $z=\left(z^{1}, \ldots, z^{n}\right)$ and $y=z / r_{i}$ to be centered at each $x_{i} \in M$; thus $\nabla K_{i}(0)=r_{i} \nabla K\left(x_{i}\right)$. Here we write $\nabla K\left(x_{i}\right)$ instead of $\nabla K(0)$ to emphasize the fact that $\nabla K$ is evaluated at different points $x_{i}$ as $i \rightarrow \infty$. We claim that this blow-up must occur at a critical point of $K$ :

Proposition 4.4. $\nabla K\left(x_{0}\right)=\lim _{i \rightarrow \infty} \nabla K\left(x_{i}\right)=0$.

Proof. Suppose this is not true. Then there exists some $j \in\{1, \ldots, n\}$ such that $\left|\partial K / \partial z^{j}\left(x_{i}\right)\right| \geq \varepsilon$ for a constant $\varepsilon$ independent of $i$. Without loss of generality we assume $j=1$. Then from inequality (13) we know that

$$
\varepsilon r_{i} \leq C\left(r_{i} v_{i}(0)^{-2 /(n-2)}+v_{i}(0)^{2 t_{i}}\right) .
$$

Therefore

$$
r_{i} \leq C v_{i}(0)^{2 t_{i}}
$$

when $v_{i}(0)^{-2 /(n-2)}$ is sufficiently small.

Once more we look at the Pohozaev identity (5) with $X=\sum_{j} y^{j} \partial / \partial y^{j}$. We divide both sides of it by $v_{i}^{2}(\bar{y})$ so that it becomes

$$
\frac{n-2}{2 n} \frac{1}{v_{i}^{2}(\bar{y})} \int_{B_{\sigma}} X\left(R_{i}\right) d v_{g_{i}}=\frac{1}{v_{i}^{2}(\bar{y})} \int_{\partial B_{\sigma}} T_{i}\left(X, v_{i}\right) d \Sigma_{i} .
$$

Its right side is

$$
\begin{aligned}
& \frac{1}{v_{i}^{2}(\bar{y})} \int_{\partial B_{\sigma}} T_{i}\left(X, v_{i}\right) d \Sigma_{i} \\
&=\frac{1}{v_{i}^{2}(\bar{y})} \int_{\partial B_{\sigma}}\left(\operatorname{Ric}\left(g_{i}\right)-n^{-1} R\left(g_{i}\right) g_{i}\right)\left(X, v_{i}\right) d \Sigma_{i} \\
&=\frac{1}{v_{i}^{2}(\bar{y})} \int_{\partial B_{\sigma}}\left(\operatorname{Ric}\left(\left(\lambda_{i} v_{i}\right)^{4 /(n-2)} d y \otimes d y\right)\right. \\
&\left.-n^{-1} R\left(\left(\lambda_{i} v_{i}\right)^{4 /(n-2)} d y \otimes d y\right)\left(\lambda_{i} v_{i}\right)^{4 /(n-2)} d y \otimes d y\right)\left(X, v_{0}\right)\left(\lambda_{i} v_{i}\right)^{2} d \Sigma_{\sigma} \\
&= \int_{\partial B_{\sigma}}\left(\frac{\lambda_{i} v_{i}}{v_{i}(\bar{y})}\right)^{2}\left(\operatorname{Ric}\left(\left(\frac{\lambda_{i} v_{i}}{v_{i}(\bar{y})}\right)^{4 /(n-2)} d y \otimes d y\right)\right. \\
&\left.-n^{-1} R\left(\left(\frac{\lambda_{i} v_{i}}{v_{i}(\bar{y})}\right)^{4 /(n-2)} d y \otimes d y\right)\left(\frac{\lambda_{i} v_{i}}{v_{i}(\bar{y})}\right)^{4 /(n-2)} d y \otimes d y\right)\left(X, v_{0}\right) d \Sigma_{\sigma},
\end{aligned}
$$

where $v_{0}=\sigma^{-1} \sum_{j} y^{j} \partial / \partial y^{j}$ is the unit outer normal on $\partial B_{\sigma}$ with respect to the Euclidean metric $d y \otimes d y$. 
When $i \rightarrow \infty, \lambda_{i}(y)=\lambda\left(r_{i} y\right) \rightarrow \lambda\left(x_{0}\right)$ for $|y|=\sigma$. Thus when $i$ goes to $\infty$, (16) converges (up to a constant) to

$\int_{\partial B_{\sigma}} h^{2}\left(\operatorname{Ric}\left(h^{4 /(n-2)} d y \otimes d y\right)\right.$

$$
\left.-n^{-1} R\left(h^{4 /(n-2)} d y \otimes d y\right) h^{4 /(n-2)} d y \otimes d y\right)\left(X, v_{0}\right) d \Sigma_{\sigma}
$$$$
=\int_{\partial B_{\sigma}} h^{2} \cdot(n-2) h^{2 /(n-2)}\left(\operatorname{Hess}\left(h^{-2 /(n-2)}\right)\left(X, v_{0}\right)\right.
$$$$
\left.-\frac{1}{n} \Delta\left(h^{-2 /(n-2)}\right)\left\langle X, v_{0}\right\rangle\right) d \Sigma_{\sigma}
$$

(17) $=(n-2) \sigma^{-1} \int_{\partial B_{\sigma}} h^{2(n-1) /(n-2)} \cdot\left(\operatorname{Hess}\left(h^{-2 /(n-2)}\right)(X, X)\right.$

$$
\left.-\frac{1}{n} \Delta\left(h^{-2 /(n-2)}\right) \sigma^{2}\right) d \Sigma_{\sigma}
$$

We know that

$h^{-2 /(n-2)}=\left(\frac{1}{2}\left(1+|y|^{2-n}\right)\right)^{-2 /(n-2)}=2^{2 /(n-2)}|y|^{2}-\frac{2^{n /(n-2)}}{n-2}|y|^{n}+O\left(|y|^{2(n-1)}\right)$,

and by direct computation,

$$
\begin{aligned}
& \operatorname{Hess}\left(2^{2 /(n-2)}|y|^{2}-\frac{2^{n /(n-2)}}{n-2}|y|^{n}\right)(X, X)-\frac{1}{n} \Delta\left(2^{2 /(n-2)}|y|^{2}-\frac{2^{n /(n-2)}}{n-2}|y|^{n}\right) \sigma^{2} \\
&=-2^{n /(n-2)}(n-1) \sigma^{n} .
\end{aligned}
$$

Therefore

$\operatorname{Hess}\left(h^{-2 /(n-2)}\right)(X, X)-\frac{1}{n} \Delta\left(h^{-2 /(n-2)}\right) \sigma^{2}=-2^{n /(n-2)}(n-1) \sigma^{n}+O\left(\sigma^{2(n-1)}\right)$.

Also we know

$$
h^{2(n-1) /(n-2)}=\left(\frac{1}{2}\right)^{2(n-1) /(n-2)}|y|^{-2(n-1)}\left(1+O\left(|y|^{n-2}\right)\right) .
$$

Thus we can conclude that (17) is equal to

$$
\begin{array}{r}
-\frac{1}{2}(n-1)(n-2) \sigma^{-1} \int_{\partial B_{\sigma}}\left(|y|^{-2(n-1)}+O\left(|y|^{-n}\right)\right) \\
\left(|y|^{n}+O\left(|y|^{2(n-1)}\right)\right) \sigma^{n-1} d \Sigma_{1} \\
=-\frac{1}{2}(n-1)(n-2)+O\left(\sigma^{n-2}\right) .
\end{array}
$$

Therefore the limit of the right side of (15) is strictly less than 0 when we choose $\sigma$ to be sufficiently small.

On the other hand, the left side of (15) is

$$
\frac{n-2}{2 n} c(n)^{-1} \frac{1}{v_{i}^{2}(\bar{y})} \int_{B_{\sigma}} X\left(K_{i} v_{i}^{-\delta_{i}}\right)\left(\lambda_{i} v_{i}\right)^{2 n /(n-2)} d y .
$$


We write

$$
\begin{aligned}
& \frac{1}{v_{i}^{2}(\bar{y})} \int_{B_{\sigma}} X\left(K_{i} v_{i}^{-\delta_{i}}\right)\left(\lambda_{i} v_{i}\right)^{2 n /(n-2)} d y \\
& =\frac{1}{v_{i}^{2}(\bar{y})} \int_{B_{\sigma}} X\left(K_{i}\right) v_{i}^{p_{i}+1} \lambda_{i}^{2 n /(n-2)} d y-\frac{\delta_{i}}{v_{i}^{2}(\bar{y})} \int_{B_{\sigma}} K_{i} \lambda_{i}^{2 n /(n-2)} v_{i}^{p_{i}} X\left(v_{i}\right) d y .
\end{aligned}
$$

The second term of (18) is equal to

$$
\begin{aligned}
& -\frac{\delta_{i}}{p_{i}+1} \frac{1}{v_{i}^{2}(\bar{y})} \int_{B_{\sigma}} K_{i} \lambda_{i}^{2 n /(n-2)} X\left(v_{i}^{p_{i}+1}\right) d y \\
= & -\frac{\delta_{i}}{p_{i}+1} \frac{1}{v_{i}^{2}(\bar{y})} \int_{B_{\sigma}}\left(\operatorname{div}\left(K_{i} \lambda_{i}^{2 n /(n-2)} v_{i}^{p_{i}+1} X\right)-K_{i} \lambda_{i}^{2 n /(n-2)} v_{i}^{p_{i}+1} \operatorname{div} X\right. \\
& \left.\quad-\lambda_{i}^{2 n /(n-2)} v_{i}^{p_{i}+1} X\left(K_{i}\right)-K_{i} v_{i}^{p_{i}+1} X\left(\lambda_{i}^{2 n /(n-2)}\right)\right) d y \\
= & -\frac{\delta_{i}}{p_{i}+1} \frac{\sigma}{v_{i}^{2}(\bar{y})} \int_{\partial B_{\sigma}} K_{i} \lambda_{i}^{2 n /(n-2)} v_{i}^{p_{i}+1} d \Sigma_{\sigma} \\
& +\frac{\delta_{i}}{p_{i}+1} \frac{1}{v_{i}^{2}(\bar{y})} \int_{B_{\sigma}} K_{i} \lambda_{i}^{2 n /(n-2)} v_{i}^{p_{i}+1}\left(n+X\left(\ln K_{i}\right)+2 n /(n-2) X\left(\ln \lambda_{i}\right)\right) d y .
\end{aligned}
$$

On $\partial B_{\sigma}$, we know $v_{i} / v_{i}(\bar{y}) \rightarrow h(\sigma)$ and $v_{i} \rightarrow 0$ uniformly, so

$$
\frac{1}{v_{i}^{2}(\bar{y})} \int_{\partial B_{\sigma}} K_{i} \lambda_{i}^{2 n /(n-2)} v_{i}^{p_{i}+1} d \Sigma_{\sigma}=\int_{\partial B_{\sigma}} K_{i} \lambda_{i}^{2 n /(n-2)}\left(\frac{v_{i}}{v_{i}(\bar{y})}\right)^{2} v_{i}^{p_{i}-1} d \Sigma_{\sigma} \rightarrow 0 .
$$

Since $X=r \partial / \partial r$ and $\left|\partial\left(\ln K_{i}\right) / \partial r\right|$ and $\left|\partial\left(\ln \lambda_{i}\right) / \partial r\right|$ are uniformly bounded, we can choose $\sigma$ to be small enough (independent of $i)$ to make $n+X\left(\ln K_{i}\right)+$ $2 n /(n-2) X\left(\ln \lambda_{i}\right)>0$. Thus when $i \rightarrow \infty$, the limit of the second term of (18) is greater than or equal to 0 .

Next we will show that the limit of the first term of (18) is 0 , or equivalently,

$$
\lim _{i \rightarrow \infty} v_{i}^{2}(0) \int_{B_{\sigma}} X\left(K_{i}\right) v_{i}^{p_{i}+1} \lambda_{i}^{2 n /(n-2)} d y=0
$$

since $v_{i}(\bar{y}) \geq C v_{i}(0)^{-1}$ by Proposition 4.1. This then will end the proof because it implies that the limit of the left hand side of (15) is greater than or equal to 0 , contradicting the sign of the right hand side.

Note that

$$
\begin{aligned}
X\left(K_{i}\right)(y) & =\left(\sum_{j} y^{j} \frac{\partial K_{i}}{\partial y^{j}}\right)(y) \\
& =\left(\sum_{j} y^{j} \frac{\partial K_{i}}{\partial y^{j}}\right)(0)+\sum_{k} \frac{\partial}{\partial y^{k}}\left(\sum_{j} y^{j} \frac{\partial K_{i}}{\partial y^{j}}\right)(\varsigma) y^{k} \text { for some }|\varsigma| \leq|y| \\
& =\sum_{j} \frac{\partial K_{i}}{\partial y^{j}}(\varsigma) y^{j}+\sum_{j, k} \frac{\partial^{2} K_{i}}{\partial y^{k} \partial y^{j}}(\varsigma) \varsigma^{j} y^{k} .
\end{aligned}
$$


Therefore

$$
\begin{aligned}
& v_{i}^{2}(0)\left|\int_{B_{\sigma}} X\left(K_{i}\right) v_{i}^{p_{i}+1} \lambda_{i}^{2 n /(n-2)} d y\right| \\
& \leq v_{i}^{2}(0) \int_{B_{\sigma}} \sum_{j}\left|\frac{\partial K_{i}}{\partial y^{j}}(\varsigma)\right||y| v_{i}^{p_{i}+1} \lambda_{i}^{2 n /(n-2)} d y \\
& +v_{i}^{2}(0) \int_{B_{\sigma}} \sum_{j, k}\left|\frac{\partial^{2} K_{i}}{\partial y^{j} \partial y^{k}}(\varsigma)\right||y|^{2} v_{i}^{p_{i}+1} \lambda_{i}^{2 n /(n-2)} d y \\
& \leq C v_{i}^{2}(0) r_{i} \int_{B_{\sigma}}|y| v_{i}^{p_{i}+1} d y+C v_{i}^{2}(0) r_{i}^{2} \int_{B_{\sigma}}|y|^{2} v_{i}^{p_{i}+1} d y \\
& \leq C v_{i}^{2}(0) r_{i} \cdot v_{i}(0)^{-2 /(n-2)+(n-1) \delta_{i} / 2}+C v_{i}^{2}(0) r_{i}^{2} \cdot v_{i}(0)^{-4 /(n-2)+n \delta_{i} / 2}
\end{aligned}
$$

(by Lemma 4.2)

$\leq C v_{i}(0)^{2+2 t_{i}-2 /(n-2)}+C v_{i}(0)^{2+4 t_{i}-4 /(n-2)}$

By the definition of $t_{i}$,

$$
\lim _{i \rightarrow \infty} t_{i}=\lim _{i \rightarrow \infty}\left(1-\frac{\left(p_{i}-1\right) l_{i}}{2}\right)=1-\frac{2}{n-2} \lim _{i \rightarrow \infty} l_{i}<1-\frac{2}{n-2} \cdot \frac{2 n-5}{2}=\frac{3-n}{n-2} .
$$

Thus

$$
\lim _{i \rightarrow \infty}\left(2+2 t_{i}-\frac{2}{n-2}\right)<2+2 \cdot \frac{3-n}{n-2}-\frac{2}{n-2}=0
$$

and

$$
\lim _{i \rightarrow \infty}\left(2+4 t_{i}-\frac{4}{n-2}\right)<2+4 \cdot \frac{3-n}{n-2}-\frac{4}{n-2}=\frac{4-2 n}{n-2}<0 .
$$

Since these are all strict inequalities, we know that

$$
\lim _{i \rightarrow \infty}\left(C v_{i}(0)^{2+2 t_{i}-2 /(n-2)}+C v_{i}(0)^{2+4 t_{i}-4 /(n-2)}\right)=0,
$$

and consequently

$$
\lim _{i \rightarrow \infty} v_{i}^{2}(0)\left|\int_{B_{\sigma}} X\left(K_{i}\right) v_{i}^{p_{i}+1} \lambda_{i}^{2 n /(n-2)} d y\right|=0 .
$$

4.5. Refined estimates for $\delta_{i}$ and $\left|\nabla K_{i}\right|$. Now because $x_{0}=\lim _{i \rightarrow \infty} x_{i}$ is a critical point of the function $K$, which satisfies the flatness condition $(*)$, we have $\left|\nabla^{p} K\left(x_{i}\right)\right| \leq C_{0}\left|\nabla K\left(x_{i}\right)\right|^{(n-2-p) /(n-3)}$ when $2 \leq p \leq n-3$. When $p=2$, this implies, because $g=\lambda^{4 /(n-2)} d z^{2}$, that

$$
\left|\nabla^{2} K\left(\frac{\partial}{\partial z^{l_{1}}}, \frac{\partial}{\partial z^{l_{2}}}\right)\left(x_{i}\right)\right|=\left|\frac{\partial^{2} K}{\partial z^{l_{1}} \partial z^{l_{2}}}\left(x_{i}\right)-\Gamma_{l_{1} l_{2}}^{l}\left(x_{i}\right) \frac{\partial K}{\partial z^{l}}\left(x_{i}\right)\right| \leq C\left|\nabla K\left(x_{i}\right)\right|^{\frac{n-4}{n-3}},
$$

where $l_{1}, l_{2}, l=1,2, \ldots, n$. Therefore

$$
\left|\frac{\partial^{2} K}{\partial z^{l_{1}} \partial z^{l_{2}}}\left(x_{i}\right)\right| \leq C\left|\nabla K\left(x_{i}\right)\right|+C\left|\nabla K\left(x_{i}\right)\right|^{(n-4) /(n-3)} \leq C\left|\nabla K\left(x_{i}\right)\right|^{(n-4) /(n-3)},
$$


since $\left|\nabla K\left(x_{i}\right)\right|<1$ for sufficiently large $i$. That is,

$$
\left|\partial^{\alpha} K / \partial z^{\alpha}\left(x_{i}\right)\right| \leq C\left|\nabla K\left(x_{i}\right)\right|^{(n-2-|\alpha|) /(n-3)}
$$

for $|\alpha|=2$. Here we have used the notations that $\alpha=\left(\alpha_{1}, \alpha_{2}, \ldots, \alpha_{n}\right)$ with each $\alpha_{i} \geq 0,|\alpha|=\alpha_{1}+\alpha_{2}+\cdots+\alpha_{n}$, and

$$
\frac{\partial^{\alpha} K}{\partial z^{\alpha}}=\frac{\partial^{\alpha_{1}} \partial^{\alpha_{2}} \cdots \partial^{\alpha_{n}} K}{\left(\partial z^{1}\right)^{\alpha_{1}}\left(\partial z^{2}\right)^{\alpha_{2}} \cdots\left(\partial z^{n}\right)^{\alpha_{n}}} .
$$

Generally, when $2 \leq p<q \leq n-3$, we have

$$
\left|\nabla K\left(x_{i}\right)\right|^{(n-2-p) /(n-3)}<\left|\nabla K\left(x_{i}\right)\right|^{(n-2-q) /(n-3)},
$$

so by similar computations we have

$$
\left|\frac{\partial^{\alpha} K}{\partial z^{\alpha}}\left(x_{i}\right)\right| \leq C\left|\nabla K\left(x_{i}\right)\right|^{(n-2-|\alpha|) /(n-3)} \quad \text { for } 2 \leq|\alpha| \leq n-3 .
$$

Then since $K_{i}(y)=K\left(r_{i} y\right)$,

$$
\left|\frac{\partial^{\alpha} K_{i}}{\partial y^{\alpha}}(0)\right|=r_{i}^{|\alpha|}\left|\frac{\partial^{\alpha} K}{\partial z^{\alpha}}\left(x_{i}\right)\right| \quad \text { and } \quad\left|\nabla K_{i}(0)\right|=r_{i}\left|\nabla K\left(x_{i}\right)\right| .
$$

Thus

$$
\begin{aligned}
\left|\frac{\partial^{\alpha} K_{i}}{\partial y^{\alpha}}(0)\right| & \leq r_{i}^{|\alpha|} C\left|\nabla K\left(x_{i}\right)\right|^{(n-2-|\alpha|) /(n-3)} \\
& =C r_{i}^{(|\alpha|-1)(n-2) /(n-3)}\left|\nabla K_{i}(0)\right|^{(n-2-|\alpha|) /(n-3)} \\
& <C r_{i}\left|\nabla K_{i}(0)\right|^{(n-2-|\alpha|) /(n-3)},
\end{aligned}
$$

where the last step follows from the fact that $(|\alpha|-1)(n-2) /(n-3)>1$ and $r_{i}<1$. With this flatness condition on $K_{i}$, we can refine the estimates for $\delta_{i}$ and $\left|\nabla K_{i}\right|$ as follows.

Inequality (9) gives

$$
\begin{aligned}
\delta_{i} & \leq C\left(v_{i}(0)^{2 t_{i}}+\left|\int_{B_{\sigma}} \frac{\partial K_{i}}{\partial r}\right| y\left|v_{i}^{p_{i}+1} \lambda_{i}^{2 n /(n-2)} d y\right|\right) \\
& =C\left(v_{i}(0)^{2 t_{i}}+\left|\int_{B_{\sigma}} r \frac{\partial K_{i}}{\partial r} v_{i}^{p_{i}+1} \lambda_{i}^{2 n /(n-2)} d y\right|\right) .
\end{aligned}
$$

We write $r \partial K_{i} / \partial r=\sum_{j} y^{j} \partial K_{i} / \partial y^{j}$. For each $j=1, \ldots, n$,

$$
\begin{aligned}
\frac{\partial K_{i}}{\partial y^{j}}(y)= & \frac{\partial K_{i}}{\partial y^{j}}(0)+\sum_{|\beta|=1} \frac{\partial^{\beta}}{\partial y^{\beta}} \frac{\partial K_{i}}{\partial y^{j}}(0) y^{\beta}+\frac{1}{2 !} \sum_{|\beta|=2} \frac{\partial^{\beta}}{\partial y^{\beta}} \frac{\partial K_{i}}{\partial y^{j}}(0) y^{\beta}+\cdots \\
& +\frac{1}{(n-4) !} \sum_{|\beta|=n-4} \frac{\partial^{\beta}}{\partial y^{\beta}} \frac{\partial K_{i}}{\partial y^{j}}(0) y^{\beta}+\frac{1}{(n-3) !} \sum_{|\beta|=n-3} \frac{\partial^{\beta}}{\partial y^{\beta}} \frac{\partial K_{i}}{\partial y^{j}}(\varsigma) y^{\beta},
\end{aligned}
$$


where $|\zeta| \leq|y|$, and $y^{\beta}=y_{1}^{\beta_{1}} y_{2}^{\beta_{2}} \cdots y_{n}^{\beta_{n}}$ for $\beta=\left(\beta_{1}, \beta_{2}, \ldots, \beta_{n}\right)$. Therefore

$$
\begin{aligned}
\int_{B_{\sigma}}\left|r \frac{\partial K_{i}}{\partial r}\right| v_{i}^{p_{i}+1} \lambda_{i}^{2 n /(n-2)} d y & \\
\leq C\left(\int_{B_{\sigma}}\left|\frac{\partial K_{i}}{\partial y^{j}}(0)\right||y| v_{i}^{p_{i}+1} d y\right. & +\left.\sum_{|\beta|=1}^{n-4} \int_{B_{\sigma}} \frac{\partial^{\beta}}{\partial y^{\beta}} \frac{\partial K_{i}}{\partial y^{j}}(0)|| y\right|^{|\beta|+1} v_{i}^{p_{i}+1} d y \\
& \left.+\sum_{|\beta|=n-3} \int_{B_{\sigma}}\left|\frac{\partial^{\beta}}{\partial y^{\beta}} \frac{\partial K_{i}}{\partial y^{j}}(\varsigma)\right||y|^{n-2} v_{i}^{p_{i}+1} d y\right) .
\end{aligned}
$$

By Lemma 4.2 and Proposition 4.3, the first term satisfies

$$
\int_{B_{\sigma}}\left|\frac{\partial K_{i}}{\partial y^{j}}(0)\right||y| v_{i}^{p_{i}+1} d y \leq C\left|\nabla K_{i}(0)\right| v_{i}(0)^{-2 /(n-2)}
$$

and the last term has

$$
\sum_{|\beta|=n-3} \int_{B_{\sigma}}\left|\frac{\partial^{\beta}}{\partial y^{\beta}} \frac{\partial K_{i}}{\partial y^{j}}(\varsigma)\right||y|^{n-2} v_{i}^{p_{i}+1} d y \leq C r_{i}^{n-2} v_{i}(0)^{-2} .
$$

In addition, (21) gives, for any $1 \leq|\beta| \leq n-4$,

$$
\begin{aligned}
\int_{B_{\sigma}} \mid \frac{\partial^{\beta}}{\partial y^{\beta}} & \left.\frac{\partial K_{i}}{\partial y^{j}}(0)|| y\right|^{|\beta|+1} v_{i}^{p_{i}+1} d y \\
& \leq C r_{i} \int_{B_{\sigma}}\left|\nabla K_{i}(0)\right|^{(n-2-(|\beta|+1)) /(n-3)}|y|^{|\beta|+1} v_{i}^{p_{i}+1} d y \\
& =C r_{i} \int_{B_{\sigma}}\left|\nabla K_{i}(0)\right|^{(n-3-|\beta|) /(n-3)}|y|^{|\beta|} \cdot|y| v_{i}^{p_{i}+1} d y \\
& \leq C r_{i} \int_{B_{\sigma}}\left(\left|\nabla K_{i}(0)\right|^{\frac{n-3-|\beta|}{n-3}} \cdot \frac{n-3}{n-3-|\beta|}+\left.|y|\right|^{|\beta| \cdot \frac{n-3}{|\beta|}}\right) \cdot|y| v_{i}^{p_{i}+1} d y
\end{aligned}
$$

(by Young's inequality)

$$
\begin{aligned}
& =C r_{i}\left(\int_{B_{\sigma}}\left|\nabla K_{i}(0)\right| \cdot|y| v_{i}^{p_{i}+1} d y+\int_{B_{\sigma}}|y|^{n-2} v_{i}^{p_{i}+1} d y\right) \\
& \leq C r_{i}\left|\nabla K_{i}(0)\right| v_{i}(0)^{-2 /(n-2)}+C r_{i} v_{i}(0)^{-2} .
\end{aligned}
$$

Thus

$$
\begin{aligned}
& \int_{B_{\sigma}}\left|r \frac{\partial K_{i}}{\partial r}\right| v_{i}^{p_{i}+1} \lambda_{i}^{2 n /(n-2)} d y \\
& \leq C\left|\nabla K_{i}(0)\right| v_{i}(0)^{-2 /(n-2)} \\
& \quad+\left(C r_{i}\left|\nabla K_{i}(0)\right| v_{i}(0)^{-2 /(n-2)}+C r_{i} v_{i}(0)^{-2}\right)+C r_{i}^{n-2} v_{i}(0)^{-2} \\
& \quad \leq C\left|\nabla K_{i}(0)\right| v_{i}(0)^{-2 /(n-2)}+C r_{i} v_{i}(0)^{-2}
\end{aligned}
$$


Plugging this back into (9) we now have a refined estimate

$$
\delta_{i} \leq C\left(v_{i}(0)^{2 t_{i}}+\left|\nabla K_{i}(0)\right| v_{i}(0)^{-2 /(n-2)}+r_{i} v_{i}(0)^{-2}\right) .
$$

This will enable us to also refine the estimate for $\left|\nabla K_{i}(0)\right|$. Inequality (12) gives $\left|\int_{B_{\sigma}} \lambda_{i}^{2 n /(n-2)} v_{i}^{p_{i}+1}\left(\partial K_{i} / \partial y^{1}\right) d y\right| \leq C\left(\delta_{i} r_{i}+v_{i}(0)^{2 t_{i}}\right)$. Again we write

$$
\begin{aligned}
\frac{\partial K_{i}}{\partial y^{1}}(y)=\frac{\partial K_{i}}{\partial y^{1}}(0)+\sum_{|\beta|=1} \frac{\partial^{\beta}}{\partial y^{\beta}} \frac{\partial K_{i}}{\partial y^{1}}(0) y^{\beta}+\frac{1}{2 !} \sum_{|\beta|=2} \frac{\partial^{\beta}}{\partial y^{\beta}} \frac{\partial K_{i}}{\partial y^{1}}(0) y^{\beta}+\cdots \\
+\frac{1}{(n-4) !} \sum_{|\beta|=n-4} \frac{\partial^{\beta}}{\partial y^{\beta}} \frac{\partial K_{i}}{\partial y^{1}}(0) y^{\beta}+\frac{1}{(n-3) !} \sum_{|\beta|=n-3} \frac{\partial^{\beta}}{\partial y^{\beta}} \frac{\partial K_{i}}{\partial y^{1}}(\varsigma) y^{\beta} .
\end{aligned}
$$

Therefore we have

$$
\begin{aligned}
\int_{B_{\sigma}} \lambda_{i}^{2 n /(n-2)} v_{i}^{p_{i}+1}\left|\frac{\partial K_{i}}{\partial y^{1}}(0)\right| d y & \\
\leq\left|\int_{B_{\sigma}} \lambda_{i}^{2 n /(n-2)} v_{i}^{p_{i}+1} \frac{\partial K_{i}}{\partial y^{1}} d y\right| & +C \sum_{|\beta|=1}^{n-4} \int_{B_{\sigma}}\left|\frac{\partial^{\beta}}{\partial y^{\beta}} \frac{\partial K_{i}}{\partial y^{1}}(0)\right||y|^{|\beta|} v_{i}^{p_{i}+1} d y \\
& +C \sum_{|\beta|=n-3} \int_{B_{\sigma}}\left|\frac{\partial^{\beta}}{\partial y^{\beta}} \frac{\partial K_{i}}{\partial y^{1}}(\varsigma)\right||y|^{n-3} v_{i}^{p_{i}+1} d y \\
\leq C\left(\delta_{i} r_{i}+v_{i}(0)^{2 t_{i}}\right)+C \sum_{|\beta|=1}^{n-4} \int_{B_{\sigma}}\left|\frac{\partial^{\beta}}{\partial y^{\beta}} \frac{\partial K_{i}}{\partial y^{1}}(0)\right||y|^{|\beta|} v_{i}^{p_{i}+1} d y & +C \sum_{|\beta|=n-3} \int_{B_{\sigma}}\left|\frac{\partial^{\beta}}{\partial y^{\beta}} \frac{\partial K_{i}}{\partial y^{1}}(\varsigma)\right||y|^{n-3} v_{i}^{p_{i}+1} d y .
\end{aligned}
$$

By (8) this implies

$$
\begin{aligned}
\left|\frac{\partial K_{i}}{\partial y^{1}}(0)\right| \leq C\left(\delta_{i} r_{i}+v_{i}(0)^{2 t_{i}}\right)+C & \sum_{|\beta|=1}^{n-4} \int_{B_{\sigma}}\left|\frac{\partial^{\beta}}{\partial y^{\beta}} \frac{\partial K_{i}}{\partial y^{1}}(0)\right||y|^{|\beta|} v_{i}^{p_{i}+1} d y \\
& +C \sum_{|\beta|=n-3} \int_{B_{\sigma}}\left|\frac{\partial^{\beta}}{\partial y^{\beta}} \frac{\partial K_{i}}{\partial y^{1}}(\varsigma)\right||y|^{n-3} v_{i}^{p_{i}+1} d y .
\end{aligned}
$$

By Lemma 4.2, Proposition 4.3 and (21), we have, when $1 \leq|\beta| \leq n-4$,

$$
\begin{aligned}
\int_{B_{\sigma}} \mid \frac{\partial^{\beta}}{\partial y^{\beta}} & \left.\frac{\partial K_{i}}{\partial y^{1}}(0)|| y\right|^{|\beta|} v_{i}^{p_{i}+1} d y \\
& \leq C r_{i} \int_{B_{\sigma}}\left|\nabla K_{i}(0)\right|^{(n-2-(|\beta|+1)) /(n-3)}|y|^{|\beta|} v_{i}^{p_{i}+1} d y \\
& =\left.C r_{i} \int_{B_{\sigma}}\left|\nabla K_{i}(0)\right|^{(n-3-|\beta|) /(n-3)}|y|\right|^{|\beta|} v_{i}^{p_{i}+1} d y
\end{aligned}
$$




$$
\begin{aligned}
& \leq C r_{i} \int_{B_{\sigma}}\left(\left|\nabla K_{i}(0)\right|^{\frac{n-3-|\beta|}{n-3} \cdot \frac{n-3}{n-3-|\beta|}}+|y|^{|\beta| \cdot \frac{n-3}{|\beta|}}\right) v_{i}^{p_{i}+1} d y \\
& =C r_{i}\left(\int_{B_{\sigma}}\left|\nabla K_{i}(0)\right| v_{i}^{p_{i}+1} d y+\int_{B_{\sigma}}|y|^{n-3} v_{i}^{p_{i}+1} d y\right) \\
& \leq C r_{i}\left|\nabla K_{i}(0)\right|+C r_{i} v_{i}(0)^{-2(n-3) /(n-2)} \text {. }
\end{aligned}
$$

Furthermore,

$$
\begin{aligned}
\sum_{|\beta|=n-3} \int_{B_{\sigma}}\left|\frac{\partial^{\beta}}{\partial y^{\beta}} \frac{\partial K_{i}}{\partial y^{1}}(\varsigma)\right||y|^{n-3} v_{i}^{p_{i}+1} d y & \leq C r_{i}^{n-2} \int_{B_{\sigma}}|y|^{n-3} v_{i}^{p_{i}+1} d y \\
& \leq C r_{i}^{n-2} v_{i}(0)^{-2(n-3) /(n-2)} .
\end{aligned}
$$

Therefore

$$
\begin{aligned}
&\left|\frac{\partial K_{i}}{\partial y^{1}}(0)\right| \leq C\left(\delta_{i} r_{i}+v_{i}(0)^{2 t_{i}}\right)+\left(C r_{i}\left|\nabla K_{i}(0)\right|+C r_{i} v_{i}(0)^{-2(n-3) /(n-2)}\right)+C r_{i}^{n-2} v_{i}(0)^{-2(n-3) /(n-2)} \\
& \leq C \delta_{i} r_{i}+C v_{i}(0)^{2 t_{i}}+C r_{i}\left|\nabla K_{i}(0)\right|+C r_{i} v_{i}(0)^{-2(n-3) /(n-2)} .
\end{aligned}
$$

The same estimate also holds for $\left|\partial K_{i} / \partial y^{j}(0)\right|$, where $j=2, \ldots, n$, so we know

$$
\begin{aligned}
\left|\nabla K_{i}(0)\right| \leq & C \delta_{i} r_{i}+C v_{i}(0)^{2 t_{i}}+C r_{i}\left|\nabla K_{i}(0)\right|+C r_{i} v_{i}(0)^{-2(n-3) /(n-2)} \\
\leq & =C\left(v_{i}(0)^{2 t_{i}}+\left|\nabla K_{i}(0)\right| v_{i}(0)^{-2 /(n-2)}+r_{i} v_{i}(0)^{-2}\right) r_{i}+C v_{i}(0)^{2 t_{i}} \\
& \quad+C r_{i}\left|\nabla K_{i}(0)\right|+C r_{i} v_{i}(0)^{-2(n-3) /(n-2)}
\end{aligned}
$$

When $i$ is large enough, all the terms involving $\left|\nabla K_{i}(0)\right|$ can be absorbed into the left hand side of this inequality, therefore we get a refined estimate

$$
\begin{aligned}
\left|\nabla K_{i}(0)\right| & \leq C r_{i} v_{i}(0)^{2 t_{i}}+C r_{i}^{2} v_{i}(0)^{-2}+C v_{i}(0)^{2 t_{i}}+C r_{i} v_{i}(0)^{-2(n-3) /(n-2)} \\
& \leq C r_{i}^{2} v_{i}(0)^{-2}+C v_{i}(0)^{2 t_{i}}+C r_{i} v_{i}(0)^{-2(n-3) /(n-2)}
\end{aligned}
$$

Finally, we will prove that (19) holds. As in the proof of Proposition 4.4, this will give the desired contradiction by comparing the signs of both sides of (15), which rules out Case I. We know

$$
\begin{aligned}
v_{i}^{2}(0) & \int_{B_{\sigma}}\left|X\left(K_{i}\right)\right| v_{i}^{p_{i}+1} \lambda_{i}^{2 n /(n-2)} d y=v_{i}^{2}(0) \int_{B_{\sigma}}\left|r \frac{\partial K_{i}}{\partial r}\right| v_{i}^{p_{i}+1} \lambda_{i}^{2 n /(n-2)} d y \\
& \leq C v_{i}^{2}(0)\left(\left|\nabla K_{i}(0)\right| v_{i}(0)^{-2 /(n-2)}+r_{i} v_{i}(0)^{-2}\right) \\
& \leq C v_{i}^{2}(0)\left(\left(r_{i}^{2} v_{i}(0)^{-2}+v_{i}(0)^{2 t_{i}}+r_{i} v_{i}(0)^{-2(n-3) /(n-2)}\right) v_{i}(0)^{-2 /(n-2)}\right. \\
& \left.+r_{i} v_{i}(0)^{-2}\right) \\
& =C\left(r_{i}^{2} v_{i}(0)^{-2 /(n-2)}+v_{i}(0)^{2+2 t_{i}-2 /(n-2)}+2 r_{i}\right) .
\end{aligned}
$$


By (20) we know $\lim _{i \rightarrow \infty}\left(2+2 t_{i}-2 /(n-2)\right)<0$, and therefore

$$
\lim _{i \rightarrow \infty} v_{i}(0)^{2+2 t_{i}-2 /(n-2)}=0 .
$$

It follows from this and $\lim _{i \rightarrow \infty} r_{i}^{2} v_{i}(0)^{-2 /(n-2)}=\lim _{i \rightarrow \infty} r_{i}=0$ that

$$
\lim _{i \rightarrow \infty} v_{i}^{2}(0) \int_{B_{\sigma}}\left|X\left(K_{i}\right)\right| v_{i}^{p_{i}+1} \lambda_{i}^{2 n /(n-2)} d y=0 .
$$

This completes the proof in Case I.

\section{Ruling out Case II}

Now we consider Case II, which has been reduced to the following: There is a sequence of functions $\left\{v_{i}\right\}$, each satisfying

$$
\Delta_{g^{(i)}} v_{i}+K\left(\sigma_{i} y\right) v_{i}^{p_{i}}=0,
$$

where $\sigma_{i} \rightarrow 0$ and $g^{(i)}(y)=g_{\alpha \beta}\left(\sigma_{i} y\right) d y^{\alpha} d y^{\beta}$. The sequence $\left\{v_{i}\right\}$ has isolated blow-up point(s) $\{0, \ldots\}$.

If 0 is not a simple blow-up point, then we can do another rescaling and repeat the argument in the previous section, with $r_{i}$ replaced by $r_{i} \sigma_{i}$, to get a contradiction. Therefore 0 must be a simple blow-up point for $\left\{v_{i}\right\}$. Then we can still repeat the argument in the previous section, with $r_{i}$ replaced by $\sigma_{i}$. The only difference is in the expression of $h=\lim _{i \rightarrow \infty} v_{i}(y) / v_{i}(\bar{y})$. As shown in [Yan 2007, Section 7], because here $|y|^{2 /\left(p_{i}-1\right)} \bar{v}_{i}(|y|)$ doesn't have a second critical point at $|y|=1$, we have a different expression of $h$ : near 0 ,

$$
h(y)=c_{1}|y|^{2-n}+A+O(|y|),
$$

where $A$ is a positive constant. This positive "mass" term $A>0$ guarantees that the limit of the boundary term of the Pohozaev identity (15) is negative, that is,

$$
\lim _{i \rightarrow \infty} \frac{1}{v_{i}^{2}(\bar{y})} \int_{\partial B_{\sigma}} T_{i}\left(X, v_{i}\right) d \Sigma_{i}<0 .
$$

The other parts of the proof remain the same. Therefore Case II can also be ruled out.

Thus we have finished the proof of Theorem 1.4.

\section{References}

[Aubin 1976] T. Aubin, "Équations différentielles non linéaires et problème de Yamabe concernant la courbure scalaire”, J. Math. Pures Appl. (9) 55:3 (1976), 269-296. MR 55 \#4288 Zbl 0336.53033 [Druet 2003] O. Druet, "From one bubble to several bubbles: the low-dimensional case", J. Differential Geom. 63:3 (2003), 399-473. MR 2004h:53051 Zbl 1070.53017 
[Druet 2004] O. Druet, "Compactness for Yamabe metrics in low dimensions", Int. Math. Res. Not. 23 (2004), 1143-1191. MR 2005b:53056 Zbl 1085.53029

[Escobar and Schoen 1986] J. F. Escobar and R. M. Schoen, "Conformal metrics with prescribed scalar curvature”, Invent. Math. 86:2 (1986), 243-254. MR 88b:58030 Zbl 0628.53041

[Gilbarg and Trudinger 2001] D. Gilbarg and N. S. Trudinger, Elliptic partial differential equations of second order, Springer, Berlin, 2001. MR 2001k:35004 Zbl 1042.35002

[Li 1995] Y. Y. Li, "Prescribing scalar curvature on $S^{n}$ and related problems, I", J. Differential Equations 120:2 (1995), 319-410. MR 98b:53031 Zbl 0827.53039

[Li 1996] Y. Y. Li, "Prescribing scalar curvature on $S^{n}$ and related problems, II: Existence and compactness”, Comm. Pure Appl. Math. 49:6 (1996), 541-597. MR 98f:53036 Zbl 0849.53031

[Li and Zhang 2005] Y. Y. Li and L. Zhang, "Compactness of solutions to the Yamabe problem, II", Calc. Var. Partial Differential Equations 24:2 (2005), 185-237. MR 2006f:53049 Zbl 02220860

[Li and Zhu 1999] Y. Y. Li and M. Zhu, "Yamabe type equations on three-dimensional Riemannian manifolds", Commun. Contemp. Math. 1:1 (1999), 1-50. MR 2000m:53051 Zbl 0973.53029

[Marques 2005] F. C. Marques, "A priori estimates for the Yamabe problem in the non-locally conformally flat case”, J. Differential Geom. 71:2 (2005), 315-346. MR 2006i:53046 Zbl 05033801

[Schoen 1984] R. Schoen, "Conformal deformation of a Riemannian metric to constant scalar curvature”, J. Differential Geom. 20:2 (1984), 479-495. MR 86i:58137 Zbl 0576.53028

[Schoen 1988a] R. Schoen, "Topics in differential geometry", Lecture notes, Stanford University, 1988.

[Schoen 1988b] R. M. Schoen, "The existence of weak solutions with prescribed singular behavior for a conformally invariant scalar equation", Comm. Pure Appl. Math. 41:3 (1988), 317-392. MR 89e:58119 Zbl 0674.35027

[Schoen 1989] R. M. Schoen, "Variational theory for the total scalar curvature functional for Riemannian metrics and related topics", pp. 120-154 in Topics in calculus of variations (Montecatini Terme, 1987), edited by M. Giaquinta, Lecture Notes in Math. 1365, Springer, Berlin, 1989. MR 90g:58023 Zbl 0702.49038

[Schoen 1991] R. M. Schoen, "On the number of constant scalar curvature metrics in a conformal class", pp. 311-320 in Differential geometry, edited by B. Lawson and K. Tenenblat, Pitman Monogr. Surveys Pure Appl. Math. 52, Longman Sci. Tech., Harlow, 1991. MR 94e:53035 Zbl 0733.53021

[Trudinger 1968] N. S. Trudinger, "Remarks concerning the conformal deformation of Riemannian structures on compact manifolds", Ann. Scuola Norm. Sup. Pisa (3) 22 (1968), 265-274. MR 39 \#2093 Zbl 0159.23801

[Yan 2007] Y. Yan, "Some compactness results related to scalar curvature deformation", Commun. Contemp. Math. 9:1 (2007), 81-120. MR 2008c:35088 Zbl 05161552

Received January 24, 2007. Revised May 6, 2008.

\section{YU YAN}

DePartMent of MATHEMATICS AND COMPUTER SCIENCE

HOUGHTON COLLEGE

Houghton, NY 14744

UNITED STATES

Yu.Yan@houghton.edu 